\title{
Sequential-Based Approach for Estimating the Stress-Strength Reliability Parameter for Exponential Distribution
}

\author{
Ashkan Khalifeh ${ }^{1}$, Eisa Mahmoudi ${ }^{1}$, and Ali Dolati ${ }^{1}$ \\ ${ }^{1}$ Department of Statistics, Yazd University, Yazd, Iran \\ Received: 25/10/2018, Revision received: 15/08/2019, Published online: 26/08/2019
}

\begin{abstract}
In this paper, two-stage and purely sequential estimation procedures are considered to construct fixed-width confidence intervals for the reliability parameter under the stress-strength model when the stress and strength are independent exponential random variables with different scale parameters. The exact distribution of the stopping rule under the purely sequential procedure is approximated using the law of large numbers and Monte Carlo integration. For the two-stage sequential procedure, explicit formulas for the distribution of the total sample size, the expected value and mean squared error of the maximum likelihood estimator of the reliability parameter under the stress-strength model are provided. Moreover, it is shown that both proposed sequential procedures are finite, and in exceptional cases, the exact distribution of stopping times is degenerate distribution at the initial sample size. The performances of the proposed methodologies are investigated with the help of simulations. Finally using real data, the procedures are clearly illustrated.
\end{abstract}

Keywords. Law of Large Numbers, Purely Sequential Sampling, Stopping Rule, Twostage Sequential Sampling.

MSC: 62L15; 60G40; 62F12; 60K10.

Ashkan Khalifeh (khalifeh68@yahoo.com)

Corresponding Author: Eisa Mahmoudi (emahmoudi@yazd.ac.ir)

Ali dolati (adolati@yazd.ac.ir) 


\section{Introduction}

As mentioned in reliability literature, stress-strength models have been described as an assessment of the reliability of a component or system in terms of variables $X$ indicating stress undertaken by the system and $Y$ representing the system's strength used to endure the stress. Concerning this scenario, the system fails if the stress surpasses the strength. Thus, $R=P(X<Y)$ is the reliability of a system under the stress-strength model. Birnbaum (1956) has introduced the main idea associated with this model. Birnbaum and McCarty (1958) developed this idea using a numerical procedure. Stress-strength models have received notable attention for many years due to their applicability in diverse areas such as medicine, physics, genetics, mechanics as well as in quality control, aerospace engineering, and civil (see, e.g., Domma and Giordano (2013) and Chapter 7 in Kotz et al. (2003)).

The problem of estimating $R$ has been widely studied under various distributional assumptions on stress and strength for a variety of data types such as complete data, censored data, and data with explanatory variables, using Monte-Carlo simulation, parametric, nonparametric and Bayesian approaches. For instance, when the independent random variables $X$ and $Y$ follow the two-parameter exponential distribution, Beg (1980) obtained uniformly minimum variance unbiased estimators (UMVUE) of $R$. Kundu and Gupta (2006) proposed the maximum likelihood estimator (MLE), two bootstrap confidence intervals and Bayesian estimate of $R$ when the Weibull model holds for stress and strength. When stress and strength are normal or lognormal random variables, Chiodo (2014) discussed a Bayesian inference method for the estimation of the reliability parameter under the stress-strength model. Based on two independent samples from Weinman multivariate exponential distributions with unknown scale parameters, Cramer and Kamps (1997) obtained UMVUE of $P(X<Y)$ for both, unknown and known common location parameter, under type-II censoring. Awad et al. (1981) and Nadarajah and Kotz (2006) considered a bivariate exponential model for stress and strength.

When stress and strength follow exponential distributions, Tong (1974) derived minimum variance unbiased estimator (MVUE) of $R$, also several authors discussed evaluation of the mean square error (MSE) of MLE of $R$ (see, e.g., Kelley et al. (1976), and Chao (1982)), Moreover Enis and Geisser (1971) applied Bayesian approach to estimate $R$. Mahmoudi et al. (2018) considered minimum risk sequential point estimation of $R$ and utilized the expected sample size and risk of the sequential procedure. Comprehensive reviews of frequentist inference for stress-strength models are given in Johnson (1988). Kotz et al. (2003) have provided a comprehensive review 
of the development of the stress-strength model up to the year 2003. Another review of stress-strength models and interference models are considered by Patowary et al. (2013).

Govindarajulu (1974) has considered the sequential procedure for the problem of obtaining the confidence interval for the parameter $R$ in three cases, the first of which assumes that $(X, Y)$ follow the bivariate normal distribution and the next two apply the nonparametric approaches. Bandyopadhyay et al. (2003) considered a nonparametric fixed-width confidence interval estimation for the parameter $R$ by adopting a partial sequential sampling scheme. Recently, Mukhopadhyay and Zhuang (2016) have considered a fixed-accuracy interval for the reliability parameter under a bivariate exponential model, namely, the Fisher's Nile example. Bapat (2018) has constructed a purely sequential estimation methodology to find a fixed-accuracy confidence interval for the unknown parameter $R$ in two separate cases, where $(X, Y)$ have a bivariate exponential distribution due to Marshall and Olkin (1967) and Freund (1961).

As far as we are concerned, there has been no research on sequential analysis for constructing a fixed width confidence interval of the stress-strength reliability parameter, in which the stress and strength distributions are supposed to be exponential. A real example in the composites industry is studied, using the stress-strength model to illustrate the useful application of our proposed method. Referring to Xia et al. (2009), by using the strengths of two jute fibers possessing two different diameters, say, $10 \mathrm{~mm}$ and $20 \mathrm{~mm}$, the reliability parameter under the stress-strength model is analyzed.

In order to construct an asymptotic, fixed-width $2 d$ confidence interval for the stress-strength reliability parameter $R$ by using its MLE, we first need to assess how many samples are needed to construct a $100(1-\alpha) \%$ confidence interval. We consider two sequential procedures and seek to answer the question of whether the procedures attain a prescribed confidence level or not. Regarding this point, the rest of the article is composed of the following sections. In section 2, Asymptotic and exact confidence intervals based on a fixed sample size are introduced. Section 3 introduces a twostage sequential methodology where the fixed-width $2 d(d>0)$ confidence interval is considered. In section 4, a purely sequential procedure is defined, and some characteristics of its stopping time are discussed. Moreover, an approximation of the exact distribution of purely sequential procedure, using the law of large numbers is provided. To clarify the theoretical results of the previous sections, we give some simulations in Section 5. In section 6, using real datasets, we illustrate the useful application of our proposed method. Finally section 7 , ends with the conclusion. 


\section{Asymptotic and Exact Confidence Interval Based on a Fixed Sample Size}

In this section, the inferential procedure about the reliability parameter $R=P(X<$ $Y)$, where $X$ and $Y$ are independent, and both have exponential distributions with different scale parameters, is represented based on fixed sample sizes. An exponential distribution denoted by $\operatorname{Exp}(\theta)$ has the probability density function (pdf) given as follows:

$$
f(x ; \theta)=\frac{1}{\theta} \exp \left(-\frac{x}{\theta}\right), x>0, \theta>0,
$$

where $\theta$ is the scale parameter. Let $X \sim \operatorname{Exp}\left(\theta_{1}\right)$ be independent of $Y \sim \operatorname{Exp}\left(\theta_{2}\right)$, then the stress-strength reliability parameter can be expressed as follows:

$$
R=P(X<Y)=\frac{\theta_{2}}{\theta_{1}+\theta_{2}} .
$$

Assume that the scale parameters $\theta_{1}$ and $\theta_{2}$ are both unknown. We want to estimate stress-strength reliability parameter $R=\theta_{2} /\left(\theta_{1}+\theta_{2}\right)$. Let $X_{1}, \ldots, X_{n}$ and $Y_{1}, \ldots, Y_{n}$ be two independent random samples from $\operatorname{Exp}\left(\theta_{1}\right)$ and $\operatorname{Exp}\left(\theta_{2}\right)$, respectively. So, the MLE of $R$ is

$$
\widehat{R}_{n}=\frac{\bar{Y}_{n}}{\bar{X}_{n}+\bar{Y}_{n}}
$$

where $\bar{X}_{n}=n^{-1} \sum_{i=1}^{n} X_{i}$ and $\bar{Y}_{n}=n^{-1} \sum_{i=1}^{n} Y_{i}$. Considering the multivariate central limit theorem (CLT), the asymptotic distribution of $\left(\bar{X}_{n}, \bar{Y}_{n}\right)^{T}$ is given by

$$
\sqrt{n}\left(\left(\begin{array}{l}
\bar{X}_{n} \\
\bar{Y}_{n}
\end{array}\right)-\left(\begin{array}{l}
\theta_{1} \\
\theta_{2}
\end{array}\right)\right) \stackrel{D}{\rightarrow} N_{2}\left(\mathbf{0},\left[\begin{array}{cc}
\theta_{1}^{2} & 0 \\
0 & \theta_{2}^{2}
\end{array}\right]\right) \quad \text { as } n \longrightarrow \infty,
$$

where $N_{2}$ denotes bivariate normal distribution and $\stackrel{D}{\rightarrow}$ stands for convergence in distribution. So, the asymptotic distribution of $\hat{R}_{n}$ is (see Chapter 7 of Ferguson (1996))

$$
\sqrt{n}\left(\widehat{R}_{n}-R\right) \stackrel{D}{\rightarrow} N\left(0, \frac{2 \theta_{1}^{2} \theta_{2}^{2}}{\left(\theta_{1}+\theta_{2}\right)^{4}}\right) \text { as } n \longrightarrow \infty .
$$

Using (2.1) for a given $d>0$ and $\alpha \in(0,1)$, a confidence interval for $R$ with length $2 d$ based on $\hat{R}_{n}$ is $I_{n}=\left[\hat{R}_{n}-d, \hat{R}_{n}+d\right]$. Lim et al. (2004) gave a purely sequential fixedwidth confidence interval estimation procedure for a "smooth" function of the two scale parameters $\theta_{1}$ and $\theta_{2}$ when $d \longrightarrow 0$. Moreover for $d$ sufficiently small Govindarajulu 
(2004) provided a sequential procedure to construct a fixed-width $2 d$ confidence interval for $R$ when $(X, Y)$ has a bivariate normal distribution with an unknown mean vector and an unknown covariance matrix. In this case, if one choose $d$ so large, lower and upper limits of confidence interval $I_{n}$ might be negative or greater than 1 , respectively. So, assume that $d$ is sufficiently small $(d \longrightarrow 0)$. For the coverage probability $(\mathrm{CP})$ of $I_{n}$ with confidence at least $1-\alpha$, we should have

$$
P\left(R \in I_{n}\right)=P\left(\left|\widehat{R}_{n}-R\right| \leq d\right) \geq 1-\alpha .
$$

Let $a=Z_{1-\alpha / 2}>0$ be such that $\Phi(a)=1-\alpha / 2$ where $\Phi(\cdot)$ is standard normal cumulative distribution function. For

$$
n^{*}=\frac{2 a^{2} \theta_{1}^{2} \theta_{2}^{2}}{d^{2}\left(\theta_{1}+\theta_{2}\right)^{4}},
$$

it follows from (2.1) that once $n^{*}$ is sufficiently large, for all $n>n^{*}$,

$$
\begin{aligned}
P\left(R \in I_{n}\right) & =P\left(\left|\frac{\sqrt{n}\left(\widehat{R}_{n}-R\right)}{\sqrt{\frac{2 \theta_{1}^{2} \theta_{2}^{2}}{\left(\theta_{1}+\theta_{2}\right)^{4}}}}\right| \leq \frac{\sqrt{n} d}{\sqrt{\frac{2 \theta_{1}^{2} \theta_{2}^{2}}{\left(\theta_{1}+\theta_{2}\right)^{4}}}}\right) \\
& \geq P\left(\left|\frac{\sqrt{n}\left(\widehat{R}_{n}-R\right)}{\sqrt{\frac{2 \theta_{1}^{2} \theta_{2}^{2}}{\left(\theta_{1}+\theta_{2}\right)^{4}}}}\right| \leq a\right) \approx 1-\alpha,
\end{aligned}
$$

that is, $n^{*}$ is the optimal (smallest) fixed sample size, which approximately satisfies (2.2). The exact coverage probability of $I_{n}$ is given in the following theorem.

Theorem 2.1. Let $C P(R)=P\left(R-d \leq \hat{R}_{n} \leq R+d\right)$, then

$$
C P(R)= \begin{cases}F_{\theta_{1}, \theta_{2}}\left(\frac{1}{R-d}-1 ; n\right)-F_{\theta_{1}, \theta_{2}}\left(\frac{1}{R+d}-1 ; n\right) & R>d \\ 1-F_{\theta_{1}, \theta_{2}}\left(\frac{1}{R+d}-1 ; n\right) & R \leq d\end{cases}
$$

where $F_{\theta_{1}, \theta_{2}}(. ; n)$ is the cumulative distribution function $(C D F)$ of random variable $T_{n}=$ $\bar{X}_{n} / \bar{Y}_{n}$.

Proof. The proof involves looking at CDF of random variable $T_{n}$. Let $P o(\cdot ; \mu)$ denotes the cumulative distribution function of a Poisson distribution with mean $\mu$. Assume 
that $X \sim G(h ; 1)$ in which $G(h ; 1)$ stands for a gamma random variable with the shape parameter $h$ and scale parameter 1. The gamma-Poisson formula (see Kao (1997), p. 51 ) is given by

$$
P(X>x)=P o(h-1 ; x), \quad 0 \leq x<\infty .
$$

Let $F_{\theta_{1}, \theta_{2}}(t ; n)=P\left(T_{n} \leq t\right)$, according to the iterative expectation formula, we have

$$
\begin{aligned}
F_{\theta_{1}, \theta_{2}}(t ; n) & =P\left(\frac{\sum_{i=1}^{n} X_{i}}{\sum_{i=1}^{n} Y_{i}} \leq t\right) \\
& =E\left\{E\left[I\left\{\frac{\sum_{i=1}^{n} X_{i}}{\theta_{1}} \leq \frac{t \sum_{i=1}^{n} Y_{i}}{\theta_{1}}\right\} \mid \sum_{i=1}^{n} Y_{i}\right]\right\},
\end{aligned}
$$

where $I\{\}$ is the indicator function. Given that $\theta_{1}^{-1} \sum_{i=1}^{n} X_{i} \sim G(n, 1), \bar{X}_{n}$ and $\bar{Y}_{n}$ are independent and also using gamma-Poisson formula (2.4), we have

$$
\begin{aligned}
E\left[I\left\{\frac{\sum_{i=1}^{n} X_{i}}{\theta_{1}} \leq \frac{t \sum_{i=1}^{n} Y_{i}}{\theta_{1}}\right\} \mid \sum_{i=1}^{n} Y_{i}=y\right] & =P\left(\frac{\sum_{i=1}^{n} X_{i}}{\theta_{1}} \leq \frac{t y}{\theta_{1}}\right) \\
& =1-\sum_{j=0}^{n-1} \frac{(y t)^{j} \exp \left\{-\frac{y t}{\theta_{1}}\right\}}{\theta_{1}^{j} j !} .
\end{aligned}
$$

Therefore,

$$
\begin{aligned}
F_{\theta_{1}, \theta_{2}}(t ; n) & =1-E\left[\sum_{j=0}^{n-1} \frac{\left(t \sum_{i=1}^{n} Y_{i}\right)^{j} \exp \left\{-\frac{t \sum_{i=1}^{n} Y_{i}}{\theta_{1}}\right\}}{\theta_{1}^{j} j !}\right] \\
& =1-\sum_{j=0}^{n-1} \frac{1}{\theta_{1}^{j} j !} E\left[\left(t \sum_{i=1}^{n} Y_{i}\right)^{j} \exp \left\{-\frac{t \sum_{i=1}^{n} Y_{i}}{\theta_{1}}\right\}\right] .
\end{aligned}
$$


Now let $U=t \sum_{i=1}^{n} Y_{i}$, obviously $U \sim G\left(n, 1 /\left(t \theta_{2}\right)\right)$, so

$$
\begin{aligned}
E\left[U^{j} \exp \left\{\frac{-U}{\theta_{1}}\right\}\right] & =\int_{0}^{\infty} \frac{\left(t \theta_{2}\right)^{-n}}{\Gamma(n)} u^{n-1} \exp \left\{\frac{-u}{t \theta_{2}}\right\} u^{j} \exp \left\{\frac{-u}{\theta_{1}}\right\} d u \\
& =\int_{0}^{\infty} \frac{\left(t \theta_{2}\right)^{-n}}{\Gamma(n)} u^{n+j-1} \exp \left\{-u\left(\frac{1}{\theta_{1}}+\frac{1}{t \theta_{2}}\right)\right\} d u \\
& =\frac{\Gamma(n+j)}{\Gamma(n)}\left(t \theta_{2}\right)^{-n}\left(\frac{t \theta_{1} \theta_{2}}{t \theta_{2}+\theta_{1}}\right)^{n+j} \\
& =\frac{(n+j-1) !}{(n-1) !}\left(\frac{t \theta_{1} \theta_{2}}{t \theta_{2}+\theta_{1}}\right)^{j}\left(\frac{\theta_{1}}{t \theta_{2}+\theta_{1}}\right)^{n}
\end{aligned}
$$

Hence, for $t>0$

$$
\begin{aligned}
F_{\theta_{1}, \theta_{2}}(t ; n) & =1-\sum_{j=0}^{n-1} \frac{(n+j-1) !}{\theta_{1}^{j} j !(n-1) !}\left(\frac{t \theta_{1} \theta_{2}}{t \theta_{2}+\theta_{1}}\right)^{j}\left(\frac{\theta_{1}}{t \theta_{2}+\theta_{1}}\right)^{n} \\
& =1-\sum_{j=0}^{n-1}\left(\begin{array}{c}
n+j-1 \\
n-1
\end{array}\right)\left(\frac{t \theta_{2}}{t \theta_{2}+\theta_{1}}\right)^{j}\left(\frac{\theta_{1}}{t \theta_{2}+\theta_{1}}\right)^{n},
\end{aligned}
$$

equals 0 for $t \leq 0$. Remember that $T_{n}$ is a positive random variable. On the other hand,

$$
\begin{aligned}
C P(R) & =P\left(R-d \leq \hat{R}_{n} \leq R+d\right) \\
& =P\left(R-d \leq \frac{1}{1+\frac{\bar{X}_{n}}{\bar{Y}_{n}}} \leq R+d\right) \\
& = \begin{cases}P\left(T_{n} \leq \frac{1}{R-d}-1\right)-P\left(T_{n} \leq \frac{1}{R+d}-1\right) & R>d \\
1-P\left(T_{n} \leq \frac{1}{R+d}-1\right) & R \leq d,\end{cases}
\end{aligned}
$$

which completes the proof.

CDF of the random variable $T_{n}$ and Theorem 2.1 will come into use in the next section and along with defining stopping rule (2.5). The required sample size to obtain an exact coverage probability greater than $1-\alpha$ is

$$
\tilde{n}(R)=\min \{n \geq 1 \mid C P(R) \geq 1-\alpha\} .
$$

Stopping rule (2.5), in which $\hat{R}_{n}=\bar{Y}_{n} /\left(\bar{X}_{n}+\bar{Y}_{n}\right)$ is substituted for $R$, is too complicated and is asymptotically equivalent to the classical stopping rules based on the asymptotic 
coverage probability. We often have the required coverage when $n \geq n^{*}$ also in small and medium-size samples. As an example, for $\alpha=0.05, \theta_{1}=1$ and $\theta_{2}=2$, by substituting $n^{*}$ for $n$ in (2.3), the results summarized as in Table 1 are acquired.

Table 1: Exact coverage probability for different values of $n^{*}$.

\begin{tabular}{ccc}
\hline$d$ & $n^{*}$ & $C P(R)$ \\
\hline 0.1377 & 20 & 0.9517 \\
0.0616 & 100 & 0.9503 \\
0.0436 & 200 & 0.9501 \\
0.0275 & 500 & 0.9500 \\
0.0195 & 1000 & 0.9500 \\
\hline
\end{tabular}

If $\theta_{1}$ and $\theta_{2}$ are known, consequently the asymptotically smallest sample size is known, and no sequential methods is needed to be applied. The problem of sequential estimation arises when the parameters are unknown. Therefore, two procedures, namely two-stage sequential and purely sequential procedures, are considered for this problem.

\section{Two-Stage Sequential Procedure for Fixed-Width Confidence Interval Estimation of the Stress-Strength Reliability Parame- ter}

Let $X_{1}, \ldots, X_{m}$ and $Y_{1}, \ldots, Y_{m}$ be pilot samples of $\operatorname{Exp}\left(\theta_{1}\right)$ and $\operatorname{Exp}\left(\theta_{2}\right)$, respectively. The statistical and mathematical principles relating to the two-stage procedures have been given by Stein (1945) and are used here to define the stopping rule as follows:

$$
N_{m}=\max \left\{\left\lfloor\frac{2 a^{2} \bar{X}_{m}^{2} \bar{Y}_{m}^{2}}{d^{2}\left(\bar{X}_{m}+\bar{Y}_{m}\right)^{4}}\right\rfloor+1, m\right\},
$$

where $\lfloor x\rfloor$ denotes the largest integer less than $x$. If $N_{m}=m$, we do not take any more observations at the second stage. On the other hand, if $N_{m}>m$, the initial samples do not suffice and we then sample the difference $(N-m)$ at the second stage. Finally, sampling stops with $X_{1}, \ldots, X_{N_{m}}, Y_{1}, \ldots, Y_{N_{m}}$, and interval $I_{N_{m}}=\left[\hat{R}_{N_{m}}-d, \hat{R}_{N_{m}}+d\right]$ based on all the $2 N_{m}$ samples is proposed for $R$. Our next goal is to determine the distribution of 
$N_{m}$ using CDF of $T_{n}$ and Theorem 2.1, in which the next result deals with this problem. Throughout this paper, $n$ is an integer number. For each $n<\left\lfloor a^{2} /\left(8 d^{2}\right)\right\rfloor+1, a_{n}$ and $b_{n}$ are the real-valued roots of the quadratic equation of the form $\left.x^{2}-\left(\sqrt{2 a^{2} /\left(d^{2} l\right.}\right)-2\right) x+1=0$, in which $l=n,\left(a_{n}<b_{n}\right)$.

Theorem 3.1. we have the following results:

(i) The probability mass function (PMF) of $N_{m}$, in a two-stage procedure is given by

$$
P\left(N_{m}=m\right)= \begin{cases}1-F_{\theta_{1}, \theta_{2}}\left(b_{m} ; m\right)+F_{\theta_{1}, \theta_{2}}\left(a_{m} ; m\right) & m<\left\lfloor\frac{a^{2}}{8 d^{2}}\right\rfloor+1 \\ 1 & m \geq\left\lfloor\frac{a^{2}}{8 d^{2}}\right\rfloor+1,\end{cases}
$$

(ii) If $m<\left\lfloor\frac{a^{2}}{8 d^{2}}\right\rfloor+1$, then the PMF becomes

$$
P\left(N_{m}=n\right)= \begin{cases}A_{n} & m+1 \leq n<\left\lfloor\frac{a^{2}}{8 d^{2}}\right\rfloor+1 \\ F_{\theta_{1}, \theta_{2}}\left(b_{\left\lfloor\frac{a^{2}}{8 d^{2}}\right\rfloor} ; m\right)-F_{\theta_{1}, \theta_{2}}\left(a_{\left\lfloor\frac{a^{2}}{8 d^{2}}\right\rfloor} ; m\right) & n=\left\lfloor\frac{a^{2}}{8 d^{2}}\right\rfloor+1 \\ 0 & n>\left\lfloor\frac{a^{2}}{8 d^{3}}\right\rfloor+1\end{cases}
$$

where $A_{n}=F_{\theta_{1}, \theta_{2}}\left(b_{n-1} ; m\right)+F_{\theta_{1}, \theta_{2}}\left(a_{n} ; m\right)-\left(F_{\theta_{1}, \theta_{2}}\left(b_{n} ; m\right)+F_{\theta_{1}, \theta_{2}}\left(a_{n-1} ; m\right)\right)$.

Proof. (i) Using the CDF of $T_{n}$ and stopping variable (3.1), we have

$$
\begin{aligned}
P\left(N_{m}=m\right) & =P\left(\frac{2 a^{2} \bar{X}_{m}^{2} \bar{Y}_{m}^{2}}{d^{2}\left(\bar{X}_{m}+\bar{Y}_{m}\right)^{4}} \leq m\right) \\
& =P\left(T_{m}^{2}-\left(\sqrt{\frac{2 a^{2}}{d^{2} m}}-2\right) T_{m}+1 \geq 0\right) .
\end{aligned}
$$

Let $\Delta$ be equal to the discriminant of the quadratic equation inside (3.2). If $\Delta$ is nonpositive, then $P\left(N_{m}=m\right)=1$. $\Delta$ is non-positive when $m \geq a^{2} /\left(8 d^{2}\right)$. It means $N_{m}$ has a degenerate distribution at $m$ if and only if $m \geq\left\lfloor a^{2} /\left(8 d^{2}\right)\right\rfloor+1 . \Delta$ is positive when $m<\left\lfloor a^{2} /\left(8 d^{2}\right)\right\rfloor+1$, so

$$
P\left(N_{m}=m\right)=P\left(T_{m}^{2}-\left(\sqrt{\frac{2 a^{2}}{d^{2} m}}-2\right) T_{m}+1 \geq 0\right)
$$




$$
\begin{aligned}
& =1-P\left(a_{m} \leq T_{m} \leq b_{m}\right) \\
& =1-F_{\theta_{1}, \theta_{2}}\left(b_{m} ; m\right)+F_{\theta_{1}, \theta_{2}}\left(a_{m} ; m\right) .
\end{aligned}
$$

(ii) If $m \geq\left\lfloor a^{2} /\left(8 d^{2}\right)\right\rfloor+1$, then $N_{m}$ has a degenerate distribution at initial sample size $m$ and consequently $P\left(N_{m}=n\right)=0$ for each $n \neq m$. For $m<\left\lfloor a^{2} /\left(8 d^{2}\right)\right\rfloor+1$, the proof is as follows:

$$
\begin{aligned}
P\left(N_{m}=n\right) & =P\left(n-1 \leq \frac{2 a^{2} \bar{X}_{m}^{2} \bar{Y}_{m}^{2}}{d^{2}\left(\bar{X}_{m}+\bar{Y}_{m}\right)^{4}} \leq n\right) \\
& =P\left(\frac{2 a^{2} \bar{X}_{m}^{2} \bar{Y}_{m}^{2}}{d^{2}\left(\bar{X}_{m}+\bar{Y}_{m}\right)^{4}} \leq n\right)-P\left(\frac{2 a^{2} \bar{X}_{m}^{2} \bar{Y}_{m}^{2}}{d^{2}\left(\bar{X}_{m}+\bar{Y}_{m}\right)^{4}} \leq n-1\right) \\
& =P\left(T_{m}^{2}-\left(\sqrt{\frac{2 a^{2}}{d^{2} n}}-2\right) T_{m}+1 \geq 0\right) \\
& -P\left(T_{m}^{2}-\left(\sqrt{\frac{2 a^{2}}{d^{2}(n-1)}}-2\right) T_{m}+1 \geq 0\right) .
\end{aligned}
$$

The first term on the right hand side of (3.3) is equal to 1 when $n \geq a^{2} /\left(8 d^{2}\right)$ and the second one is equal to 1 when $n \geq a^{2} /\left(8 d^{2}\right)+1$. So for each $n>\left\lfloor a^{2} /\left(8 d^{2}\right)\right\rfloor+1$, $P\left(N_{m}=n\right)=0$. Let $n=\left\lfloor a^{2} /\left(8 d^{2}\right)\right\rfloor+1$, then

$$
\begin{aligned}
P\left(N_{m}=n\right) & =P\left(T_{m}^{2}-\left(\sqrt{\frac{2 a^{2}}{d^{2} n}}-2\right) T_{m}+1 \geq 0\right) \\
& -P\left(T_{m}^{2}-\left(\sqrt{\frac{2 a^{2}}{d^{2}(n-1)}}-2\right) T_{m}+1 \geq 0\right) \\
& =1-\left[1-P\left(a_{n-1} \leq T_{m} \leq b_{n-1}\right)\right] \\
& =F_{\theta_{1}, \theta_{2}}\left(b_{n-1} ; m\right)-F_{\theta_{1}, \theta_{2}}\left(a_{n-1} ; m\right) \\
& =F_{\theta_{1}, \theta_{2}}\left(b_{\left\lfloor a^{2} /\left(8 d^{2}\right)\right\rfloor} ; m\right)-F_{\theta_{1}, \theta_{2}}\left(a_{\left\lfloor a^{2} /\left(8 d^{2}\right)\right\rfloor}\right) .
\end{aligned}
$$

Finally, for each $m+1 \leq n<\left\lfloor a^{2} /\left(8 d^{2}\right)\right\rfloor+1$,

$$
\begin{aligned}
P\left(N_{m}=n\right) & =P\left(T_{m}^{2}-\left(\sqrt{\frac{2 a^{2}}{d^{2} n}}-2\right) T_{m}+1 \geq 0\right) \\
& -P\left(T_{m}^{2}-\left(\sqrt{\frac{2 a^{2}}{d^{2}(n-1)}}-2\right) T_{m}+1 \geq 0\right)
\end{aligned}
$$




$$
\begin{aligned}
& =P\left(a_{n-1} \leq T_{m} \leq b_{n-1}\right)-P\left(a_{n} \leq T_{m} \leq b_{n}\right) \\
& =F_{\theta_{1}, \theta_{2}}\left(b_{n-1} ; m\right)+F_{\theta_{1}, \theta_{2}}\left(a_{n} ; m\right)-\left(F_{\theta_{1}, \theta_{2}}\left(b_{n} ; m\right)+F_{\theta_{1}, \theta_{2}}\left(a_{n-1} ; m\right)\right) .
\end{aligned}
$$

Theorem 3.1 summarizes by saying that, if starting sample size $m \geq\left\lfloor a^{2} /\left(8 d^{2}\right)\right\rfloor+1$, then the random variable $N_{m}$ has a degenerate distribution at the initial sample size $m$. $N_{m}$ is finite and attains values $m, \ldots,\left\lfloor a^{2} /\left(8 d^{2}\right)\right\rfloor+1$ when $m<\left\lfloor a^{2} /\left(8 d^{2}\right)\right\rfloor+1$. Stopping rule $N_{m}$ is the estimate of the unknown optimum sample size $n^{*}$, so we are interested in computing the expectation of this random variable. The next theorem deals with the expectation of the stopping rule $N_{m}$ for the case $m<\left\lfloor a^{2} /\left(8 d^{2}\right)\right\rfloor+1$, because if $m \geq\left\lfloor a^{2} /\left(8 d^{2}\right)\right\rfloor+1$, it has been proved in part (i) of Theorem 3.1 that $N_{m}$ has a degenerate distribution at $m$ and hence $E\left[N_{m}\right]=m$. The following Lemma will help us to compute the expectation of random variable $N_{m}$.

Lemma 3.1. The cumulative distribution function of $N_{m}$ is

$$
P\left(N_{m} \leq k\right)= \begin{cases}0 & k<m \\ 1-F_{\theta_{1}, \theta_{2}}\left(b_{\langle k\rangle} ; m\right)+F_{\theta_{1}, \theta_{2}}\left(a_{\langle k\rangle} ; m\right) & m \leq k<\left\lfloor\frac{a^{2}}{8 d^{2}}\right\rfloor+1 \\ 1 & k \geq\left\lfloor\frac{a^{2}}{8 d^{2}}\right\rfloor+1 .\end{cases}
$$

Where $\langle x\rangle$ denotes the greatest integer less than or equal to $x$.

Proof. If $k<m$ or $k \geq\left\lfloor a^{2} /\left(8 d^{2}\right)\right\rfloor+1$, the proof is evident and the details are avoided. For the case in which $m \leq k<\left\lfloor a^{2} /\left(8 d^{2}\right)\right\rfloor+1$, the proof is as follows:

$$
\begin{aligned}
P\left(N_{m} \leq k\right) & =\sum_{i=m}^{\langle k\rangle} P\left(N_{m}=i\right) \\
& =P\left(N_{m}=m\right)+\sum_{i=m+1}^{\langle k\rangle} P\left(N_{m}=i\right),
\end{aligned}
$$

note that $\sum_{i=m+1}^{\langle k\rangle} P\left(N_{m}=i\right)$, is a telescoping series, so we have

$$
\sum_{i=m+1}^{\langle k\rangle} P\left(N_{m}=i\right)=F_{\theta_{1}, \theta_{2}}\left(b_{m} ; m\right)-F_{\theta_{1}, \theta_{2}}\left(a_{m} ; m\right)+F_{\theta_{1}, \theta_{2}}\left(a_{\langle k\rangle} ; m\right)-F_{\theta_{1}, \theta_{2}}\left(b_{\langle k\rangle} ; m\right) .
$$


Therefore,

$$
\begin{aligned}
P\left(N_{m} \leq k\right) & =P\left(N_{m}=m\right)+\sum_{i=m+1}^{\langle k\rangle} P\left(N_{m}=i\right) \\
& =1-F_{\theta_{1}, \theta_{2}}\left(b_{\langle k\rangle} ; m\right)+F_{\theta_{1}, \theta_{2}}\left(a_{\langle k\rangle} ; m\right) .
\end{aligned}
$$

Theorem 3.2. For the case $m<\left\lfloor a^{2} /\left(8 d^{2}\right)\right\rfloor+1$, the expectation of the stopping variable $N_{m}$ is given as

$$
E\left[N_{m}\right]=m+\sum_{i=m+1}^{\left\lfloor a^{2} /\left(8 d^{2}\right)\right\rfloor+1}\left(F_{\theta_{1}, \theta_{2}}\left(b_{i-1} ; m\right)-F_{\theta_{1}, \theta_{2}}\left(a_{i-1} ; m\right)\right) .
$$

Proof. Since $N_{m}$ is a discrete random variable with support $m, m+1, \ldots,\left\lfloor a^{2} / 8 d^{2}\right\rfloor+1$, therefore,

$$
E\left[N_{m}\right]=m+\sum_{i=m+1}^{\left\lfloor a^{2} /\left(8 d^{2}\right)\right\rfloor+1} P\left(N_{m} \geq i\right) .
$$

Now using Lemma 3.1, we have

$$
P\left(N_{m}>k\right)= \begin{cases}1 & k<m \\ F_{\theta_{1}, \theta_{2}}\left(b_{\langle k\rangle} ; m\right)-F_{\theta_{1}, \theta_{2}}\left(a_{\langle k\rangle} ; m\right) & m \leq k<\left\lfloor\frac{a^{2}}{8 d^{2}}\right\rfloor+1 \\ 0 & k \geq\left\lfloor\frac{a^{2}}{8 d^{2}}\right\rfloor+1 .\end{cases}
$$

Hence,

$$
\begin{aligned}
E\left[N_{m}\right] & =m+\sum_{i=m+1}^{\left\lfloor a^{2} /\left(8 d^{2}\right)\right\rfloor+1} P\left(N_{m} \geq i\right) \\
& =m+P\left(N_{m}>m\right)+P\left(N_{m}>m+1\right)+\cdots+P\left(N_{m}>\left\lfloor a^{2} /\left(8 d^{2}\right)\right\rfloor\right) \\
& =m+\sum_{i=m+1}^{\left\lfloor a^{2} /\left(8 d^{2}\right)\right\rfloor+1}\left(F_{\theta_{1}, \theta_{2}}\left(b_{i-1} ; m\right)-F_{\theta_{1}, \theta_{2}}\left(a_{i-1} ; m\right)\right) .
\end{aligned}
$$


Theorem 3.3. The first and second moments of $\hat{R}_{N_{m}}$ are, given respectively by

$$
E\left[\widehat{R}_{N_{m}}\right]=\sum_{n=m}^{\left\lfloor a^{2} /\left(8 d^{2}\right)\right\rfloor+1} \frac{k(n, \rho)}{B(n, n)} P\left(N_{m}=n\right),
$$

where

$$
k(n, \rho)=\int_{0}^{1} \frac{t^{n}(1-t)^{n-1}}{\rho+(1-\rho) t} d t
$$

$\rho=\theta_{1} / \theta_{2}$ and $B(\cdot, \cdot)$ is Euler's integral of the first kind (also known as beta function), and

$$
E\left[\widehat{R}_{N_{m}}^{2}\right]=\sum_{n=m}^{\left\lfloor a^{2} / 8 d^{2}\right\rfloor+1}\left[\left(\frac{n(1+\rho)}{1-\rho}+1\right) \frac{k(n, \rho)}{B(n, n)}-\frac{n}{1-\rho}\right] P\left(N_{m}=n\right) .
$$

Proof. According to Sathe and Shah (1981), we have

$$
E\left[\widehat{R}_{n}\right]=\frac{k(n, \rho)}{B(n, n)}
$$

and

$$
E\left[\widehat{R}_{n}^{2}\right]=\left(\frac{n(1+\rho)}{1-\rho}+1\right) \frac{k(n, \rho)}{B(n, n)}-\frac{n}{1-\rho}
$$

So, $E\left[\hat{R}_{N_{m}}\right]$ and $E\left[\hat{R}_{N_{m}}^{2}\right]$ are given, respectively by

$$
\begin{aligned}
E\left[\widehat{R}_{N_{m}}\right] & =E\left\{E\left[\widehat{R}_{N_{m}} \mid N_{m}\right]\right\} \\
& =E\left[\frac{k\left(N_{m}, \rho\right)}{B\left(N_{m}, N_{m}\right)}\right] \\
& =\sum_{n=m}^{\left\lfloor a^{2} / 8 d^{2}\right\rfloor+1} \frac{k(n, \rho)}{B(n, n)} P\left(N_{m}=n\right),
\end{aligned}
$$

and

$$
\begin{aligned}
E\left[\widehat{R}_{N_{m}}^{2}\right] & =E\left\{E\left[\widehat{R}_{N_{m}}^{2} \mid N_{m}\right]\right\} \\
& =E\left[\left(\frac{N_{m}(1+\rho)}{1-\rho}+1\right) \frac{k\left(N_{m}, \rho\right)}{B\left(N_{m}, N_{m}\right)}-\frac{N_{m}}{1-\rho}\right]
\end{aligned}
$$




$$
=\sum_{n=m}^{\left\lfloor a^{2} /\left(8 d^{2}\right)\right\rfloor+1}\left[\left(\frac{n(1+\rho)}{1-\rho}+1\right) \frac{k(n, \rho)}{B(n, n)}-\frac{n}{1-\rho}\right] P\left(N_{m}=n\right) .
$$

Using Theorem 3.3 the MSE of $\hat{R}_{N_{m}}$ according to $R$ is given by

$$
\operatorname{MSE}\left[\widehat{R}_{N_{m}}\right]=E\left[\widehat{R}_{N_{m}}^{2}\right]-2 R E\left[\widehat{R}_{N_{m}}\right]+R^{2} .
$$

Theorem 3.4. The coverage probability $\left(\hat{R}_{N_{m}}-d, \hat{R}_{N_{m}}+d\right)$ is as follows:

$$
\begin{aligned}
C P(R)= & \sum_{n=m}^{\left\lfloor a^{2} /\left(8 d^{2}\right)\right\rfloor+1}\left(F_{\theta_{1}, \theta_{2}}\left(\frac{1}{R-d}-1 ; n\right)-F_{\theta_{1}, \theta_{2}}\left(\frac{1}{R+d}-1 ; n\right) P\left(N_{m}=n\right)\right) I\{R>d\} \\
& +\sum_{n=m}^{\left\lfloor a^{2} /\left(8 d^{2}\right)\right\rfloor+1}\left(1-F_{\theta_{1}, \theta_{2}}\left(\frac{1}{R+d}-1 ; n\right) P\left(N_{m}=n\right)\right) I\{R \leq d\} .
\end{aligned}
$$

Proof. Using Theorem (2.1) and iterative expectation formula, we have

$$
\begin{aligned}
C P(R) & =P\left(R-d \leq \hat{R}_{N_{m}} \leq R+d\right) \\
& =E\left\{E\left[I\left\{R-d \leq \hat{R}_{N_{m}} \leq R+d\right\} \mid N_{m}\right]\right\} \\
& =E\left[\left(F_{\theta_{1}, \theta_{2}}\left(\frac{1}{R-d}-1 ; N_{m}\right)-F_{\theta_{1}, \theta_{2}}\left(\frac{1}{R+d}-1 ; N_{m}\right)\right) I\{R>d\}\right. \\
& \left.+\left(1-F_{\theta_{1}, \theta_{2}}\left(\frac{1}{R+d}-1 ; N_{m}\right)\right) I\{R \leq d\}\right] \\
& =\sum_{n=m}^{\left\lfloor a^{2} / 8 d^{2}\right\rfloor+1}\left(F_{\theta_{1}, \theta_{2}}\left(\frac{1}{R-d}-1 ; n\right)-F_{\theta_{1}, \theta_{2}}\left(\frac{1}{R+d}-1 ; n\right) P\left(N_{m}=n\right)\right) I\{R>d\} \\
& +\sum_{n=m}^{\left\lfloor a^{2} / 8 d^{2}\right\rfloor+1}\left(1-F_{\theta_{1}, \theta_{2}}\left(\frac{1}{R+d}-1 ; n\right) P\left(N_{m}=n\right)\right) I\{R \leq d\} .
\end{aligned}
$$

\section{Purely Sequential-Based for Fixed-Width Confidence Interval Estimation of the Stress-Strength Reliability Parameter}

Stein's two-stage procedure ( Stein (1945)) is considered one of the most exciting and practical sequential procedure for hypotheses testing and estimation. This procedure 
estimates the required sample size, $N_{m}$ using a small number of observations as, $m$. However, the procedure tends to oversample and the amount of oversampling increases with the increase in $\left(N_{m}-m\right)$. To reduce oversampling by the two-stage procedure, we have to proceed the estimation of $R$ successively in a sequential manner. We begin with a few observations (say, $m>2$ ) and then we continue to take one additional observation at-a-time, but terminate sampling when we have gathered enough observations. As soon as a sample size exceeds the corresponding estimate of $n^{*}$, the sampling is terminated right there, and an interval estimator of $R$ is constructed. In this section, a sequential procedure for estimating the stress-strength reliability parameter for exponential distributions is proposed, and using the law of large numbers, the characteristics of its stopping time are depicted. First, note that

$$
N_{d}=\min \left\{n \geq m \mid n \geq \frac{2 a^{2} \bar{X}_{n}^{2} \bar{Y}_{n}^{2}}{d^{2}\left(\bar{X}_{n}+\bar{Y}_{n}\right)^{4}}\right\},
$$

in which $m \geq 2$ is the starting (or pilot) sample size. $N_{d}$ can be expressed in terms of $T_{n}=\bar{X}_{n} / \bar{Y}_{n}$ as follows:

$$
\begin{aligned}
N_{d} & =\min \left\{n \geq m \mid \frac{\left(\bar{X}_{n}+\bar{Y}_{n}\right)^{4}}{\bar{X}_{n}^{2} \bar{Y}_{n}^{2}} \geq \frac{2 a^{2}}{n d^{2}}\right\} \\
& =\min \left\{n \geq m \mid T_{n}^{2}-\left(\sqrt{\frac{2 a^{2}}{n d^{2}}}-2\right) T_{n}+1 \geq 0\right\} .
\end{aligned}
$$

Note that there is no real solution to the quadratic equation inside (4.2) for $n>a^{2} / 8 d^{2}$.

Theorem 4.1. Let $a_{l}$ and $b_{l}$ be the real-valued roots of the quadratic equation inside (4.2) for $n=l$, such that $a_{l}<b_{l}$, then

(i)

$$
P\left(N_{d}=m\right)= \begin{cases}1-P\left(a_{m} \leq T_{m} \leq b_{m}\right) & m<\left\lfloor\frac{a^{2}}{8 d^{2}}\right\rfloor+1 \\ 1 & m \geq\left\lfloor\frac{a^{2}}{8 d^{2}}\right\rfloor+1,\end{cases}
$$

(ii)

$$
P\left(N_{d} \leq k\right)= \begin{cases}0 & k<m \\ 1-P\left(\bigcap_{i=m}^{\langle k\rangle}\left\{a_{i} \leq T_{i} \leq b_{i}\right\}\right) & m \leq k<\left\lfloor\frac{a^{2}}{8 d^{2}}\right\rfloor+1 \\ 1 & k \geq\left\lfloor\frac{a^{2}}{8 d^{2}}\right\rfloor+1 .\end{cases}
$$


Proof. (i) The discriminant of the quadratic equation inside (4.2) is non-positive for, $n \geq a^{2} /\left(8 d^{2}\right)$. It means that for each $n \geq a^{2} /\left(8 d^{2}\right), T_{n}^{2}-\left(\sqrt{2 a^{2} /\left(n d^{2}\right)}-2\right) T_{n}+1 \geq 0$. So for each $n \geq m \geq\left\lfloor a^{2} /\left(8 d^{2}\right)\right\rfloor+1$, the quadratic equation inside (4.2) is non-negative. Therefore, the minimum $n \geq m$ such that $T_{n}^{2}-\left(\sqrt{2 a^{2} /\left(n d^{2}\right)}-2\right) T_{n}+1 \geq 0$ is $m$ itself. Thus if $m \geq\left\lfloor a^{2} /\left(8 d^{2}\right)\right\rfloor+1$, then $P\left(N_{d}=m\right)=1$ and if $m<\left\lfloor a^{2} /\left(8 d^{2}\right)\right\rfloor+1$,

$$
\begin{aligned}
P\left(N_{d}=m\right) & =P\left(T_{m}^{2}-\left(\sqrt{\frac{2 a^{2}}{m d^{2}}}-2\right) T_{m}+1 \geq 0\right) \\
& =1-P\left(a_{m} \leq T_{m} \leq b_{m}\right) .
\end{aligned}
$$

(ii) It is apparent that $P\left(N_{d} \leq k\right)=0$ if $k<m$. For $m \leq k<\left\lfloor a^{2} /\left(8 d^{2}\right)\right\rfloor+1$,

$$
\begin{aligned}
P\left(N_{d}>k\right) & =P\left(N_{d} \neq m, \ldots, N_{d} \neq k\right) \\
& =P\left(\bigcap_{i=m}^{\langle k\rangle}\left\{T_{i}^{2}-\left(\sqrt{\frac{2 a^{2}}{i d^{2}}}-2\right) T_{n}+1<0\right\}\right) \\
& =P\left(\bigcap_{i=m}^{\langle k\rangle}\left\{a_{i} \leq T_{i} \leq b_{i}\right\}\right) .
\end{aligned}
$$

Finally, if $k=\left\lfloor a^{2} /\left(8 d^{2}\right)\right\rfloor+1$, in the view of the fact that for each $n \geq a^{2} /\left(8 d^{2}\right), T_{n}^{2}-$ $\left(\sqrt{2 a^{2} /\left(n d^{2}\right)}-2\right) T_{n}+1 \geqslant 0$ (see part $(i)$ ), then we have

$$
\begin{aligned}
P\left(N_{d} \leq\left\lfloor\frac{a^{2}}{8 d^{2}}\right\rfloor+1\right) & =1-P\left(N_{d}>\left\lfloor\frac{a^{2}}{8 d^{2}}\right\rfloor+1\right) \\
& =1-P\left(N_{d} \neq m, \ldots, N_{d} \neq\left\lfloor\frac{a^{2}}{8 d^{2}}\right\rfloor+1\right) \\
& \left.=1-P\left(\bigcap_{i=m}^{\left\lfloor\frac{a^{2}}{8 d^{2}}\right\rfloor+1}\left\{T_{i}^{2}-\left(\sqrt{\frac{2 a^{2}}{i d^{2}}}-2\right) T_{n}+1<0\right\}\right)\right) \\
& =1 .
\end{aligned}
$$

So, if $k=\left\lfloor a^{2} /\left(8 d^{2}\right)\right\rfloor+1$ then $T_{k}^{2}-\left(\sqrt{2 a^{2} /\left(k d^{2}\right)}-2\right) T_{k}+1 \geqslant 0$, the intersection is an empty set, which is the desired result.

Part (i) of Theorem 4.1 shows that if the starting sample size $m \geq\left\lfloor a^{2} /\left(8 d^{2}\right)\right\rfloor+1$, then $N_{d}$ has a degenerate distribution at $m$. Part (ii) of Theorem 4.1 indicates that $N_{d}$ can 
attain values $m, m+1, \ldots,\left\lfloor a^{2} /\left(8 d^{2}\right)\right\rfloor+1$, proving that not only $P\left(N_{d}<\infty\right)=1$ but also $P\left(N_{d} \leq\left\lfloor a^{2} /\left(8 d^{2}\right)\right\rfloor+1\right)=1$.

We can approximate $P\left(N_{d}>k\right)$, by using the law of large numbers. Using part (ii) of Theorem 4.1, for each $m \leq k<\left\lfloor a^{2} /\left(8 d^{2}\right)\right\rfloor+1$, we have

$$
\begin{aligned}
P\left(N_{d}>k\right) & =P\left(\bigcap_{i=m}^{\langle k\rangle}\left\{a_{i} \leq T_{i} \leq b_{i}\right\}\right) \\
& =E\left[I\left\{\bigcap_{i=m}^{\langle k\rangle}\left\{a_{i} \leq T_{i} \leq b_{i}\right\}\right] .\right.
\end{aligned}
$$

In order to apply the strong law of large numbers, we only need to show that $E\left[\mid I\left\{\bigcap_{i=m}^{\langle k\rangle}\left\{a_{i} \leq T_{i} \leq b_{i}\right\} \mid\right]<\infty\right.$, which in this case is trivial because $\left|I\left\{\bigcap_{i=m}^{\langle k\rangle}\left\{a_{i} \leq T_{i} \leq b_{i}\right\}\right\}\right| \leq$ 1. It may be observed that, generating random numbers from the joint distribution of $\left(T_{m}, \ldots, T_{\langle k\rangle}\right)$ is very straightforward by using the definition of $\left(T_{m}, \ldots, T_{\langle k\rangle}\right)$. More precisely, first generate $X_{1}, \ldots, X_{\langle k\rangle}$ and $Y_{1}, \ldots, Y_{\langle k\rangle}$ from $\operatorname{Exp}\left(\theta_{1}\right)$ and $\operatorname{Exp}\left(\theta_{2}\right)$ distributions, respectively. Then set

$$
T_{j}=\frac{\sum_{i=1}^{j} X_{i}}{\sum_{i=1}^{j} Y_{i}}, \quad j=m, \ldots,\langle k\rangle .
$$

Now, let $\mathbf{t}_{\mathbf{m}}=\left(t_{m 1}, \ldots, t_{m n}\right)$ be $n$ independent observations from $T_{m}$. Using Monte Carlo integration, for sufficiently large $n(n \longrightarrow \infty)$, we have

$$
\widehat{P}\left(N_{d}>k\right)=\frac{1}{n} \sum_{j=1}^{n} I\left\{\bigcap_{i=m}^{k}\left\{a_{i} \leq t_{i j} \leq b_{i}\right\}\right\} \rightarrow E\left[I\left\{\bigcap_{i=m}^{k}\left\{a_{i} \leq T_{i} \leq b_{i}\right\}\right]=P\left(N_{d}>k\right) .\right.
$$

Therefore, using part (ii) of Theorem 3.4 and Monte Carlo method, we can approximate the CDF of $N_{d}$ as follows:

$$
\widehat{P}\left(N_{d} \leq k\right)= \begin{cases}0 & k<m \\ 1-\widehat{P}\left(N_{d}>k\right) & m \leq k<\left\lfloor\frac{a^{2}}{8 d^{2}}\right\rfloor+1 \\ 1 & k \geq\left\lfloor\frac{a^{2}}{8 d^{2}}\right\rfloor+1\end{cases}
$$

So, $\widehat{P}\left(N_{d}=n\right)=\widehat{P}\left(N_{d} \leq n\right)-\widehat{P}\left(N_{d} \leq n-1\right)$. Having approximated the exact distribution of $N_{d}$, we can estimate the average stopping time, the average estimate of $R$, MSE of $\widehat{R}_{N_{d}}$ 
according to $R$ and the coverage probability of $R$. Let $\widehat{P}\left(N_{d}=n\right)$ be the approximation of $P\left(N_{d}=n\right)$, so we approximate the average stopping time, $E\left[N_{d}\right]$ by $E\left[N_{d}\right]^{*}$ as follows:

$$
E\left[N_{d}\right]^{*}=\sum_{i=m}^{\left\lfloor a^{2} / 8 d^{2}\right\rfloor+1} i \widehat{P}\left(N_{d}=i\right) .
$$

To approximate $E\left[\hat{R}_{N_{d}}\right], E\left[\hat{R}_{N_{d}}^{2}\right]$, MSE of $\hat{R}_{N_{d}}$ according to $R$ and CP, we use (3.9)(3.12), respectively, in which $N_{d}$ is substituted for $N_{m}$ and $\widehat{P}\left(N_{d}=n\right)$ is substituted for $P\left(N_{m}=n\right)$. So we have

$$
\begin{gathered}
E\left[\widehat{R}_{N_{d}}\right]^{*}=\sum_{n=m}^{\left\lfloor a^{2} /\left(8 d^{2}\right)\right\rfloor+1} \frac{k(n, \rho)}{B(n, n)} \widehat{P}\left(N_{d}=n\right), \\
E\left[\widehat{R}_{N_{d}}^{2}\right]^{*}=\sum_{n=m}^{\left\lfloor a^{2} / 8 d^{2}\right\rfloor+1}\left[\left(\frac{n(1+\rho)}{1-\rho}+1\right) \frac{k(n, \rho)}{B(n, n)}-\frac{n}{1-\rho}\right] \widehat{P}\left(N_{d}=n\right),
\end{gathered}
$$

and the MSE of $\hat{R}_{N_{d}}$ according to $R$ is

$$
\operatorname{MSE}\left(\widehat{R}_{N_{d}}\right)^{*}=E\left[\widehat{R}_{N_{d}}^{2}\right]^{*}-2 R E\left[\widehat{R}_{N_{d}}\right]^{*}+R^{2} .
$$

Finally, the CP is as follows:

$$
\begin{aligned}
C P(R)^{*}= & \sum_{n=m}^{\left\lfloor a^{2} /\left(8 d^{2}\right)\right\rfloor+1}\left(F_{\theta_{1}, \theta_{2}}\left(\frac{1}{R-d}-1 ; n\right)-F_{\theta_{1}, \theta_{2}}\left(\frac{1}{R+d}-1 ; n\right) \widehat{P}\left(N_{d}=n\right)\right) I\{R>d\} \\
& +\sum_{n=m}^{\left\lfloor a^{2} /\left(8 d^{2}\right)\right\rfloor+1}\left(1-F_{\theta_{1}, \theta_{2}}\left(\frac{1}{R+d}-1 ; n\right) \widehat{P}\left(N_{d}=n\right)\right) I\{R \leq d\}
\end{aligned}
$$

\section{Computation and Simulation Results}

We have done detailed computations to justify the results of the previous sections, but for the sake of brevity, we only present a summary here. Purely and two-stage procedures are considered when $\theta_{1}=1$ and $\theta_{2}=2,3,5$ and 7 , the confidence level is $1-\alpha=0.95$, the initial sample size $m=5,10,20$ and $d$ is chosen such that $n^{*}=20,100,200$, 
500 and 1000. For each set of values, we ran $h=10,000$ replications by letting MATLAB ("MATLAB 2018a, The MathWorks, Natick, 2018") draw samples from the preassigned exponential populations. Suppose that in the $i^{\text {th }}$ replication there are $n_{i}$ observations. Based on these data, we estimate $R$ by $\bar{Y}_{n_{i}} /\left(\bar{X}_{n_{i}}+\bar{Y}_{n_{i}}\right)$. That is, for the two stage sequential procedures; we observed $N_{m}=n_{i}$ and set $\hat{R}_{N_{m}}=\bar{Y}_{n_{i}} /\left(\bar{X}_{n_{i}}+\bar{Y}_{n_{i}}\right)$. Similarly, for the purely sequential procedures, we observed $N_{d}=n_{i}$ and set $\hat{R}_{N_{d}}=\bar{Y}_{n_{i}} /\left(\bar{X}_{n_{i}}+\bar{Y}_{n_{i}}\right)$. The distribution of $N_{d}$ for the purely sequential procedure is approximated using 10,000 replications. Table 2 represents the notations used to summarize our results.

Table 2: Index of notation that is used in Table 3-10.

\begin{tabular}{|c|c|c|c|}
\hline Sampling scheme & Notation & Formula & Explain \\
\hline \multirow{8}{*}{ Two-stage (Tables 3-6) } & $E\left[N_{m}\right]$ & (3.6) & Exact expectation of $N_{m}$ \\
\hline & $\widehat{E}\left[N_{m}\right]$ & $h^{-1} \sum_{i=1}^{h} n_{i}$ & Simulated value of $E\left[N_{m}\right]$ \\
\hline & $E\left[\widehat{R}_{N_{m}}\right]$ & (3.9) & Exact expectation of $\widehat{R}_{N_{m}}$ \\
\hline & $\widehat{E}\left[\widehat{R}_{N_{m}}\right]$ & $h^{-1} \sum_{i=1}^{h} \hat{R}_{n_{i}}$ & Simulated value of expectation of $\widehat{R}_{N_{m}}$ \\
\hline & $\operatorname{MSE}\left[\widehat{R}_{N_{m}}\right]$ & $(3.11)$ & Exact value of $M S E\left[\widehat{R}_{N_{m}}\right]$ according to $R$ \\
\hline & $\widehat{M S E}$ & $h^{-1} \sum_{i=1}^{h}\left(\hat{R}_{n_{i}}-R\right)^{2}$ & Simulated value of $M S E\left[\widehat{R}_{N_{m}}\right]$ according to $R$ \\
\hline & $C P$ & $(3.12)^{l-1}$ & Exact value of coverage probability of $R$ \\
\hline & $\widehat{C P}$ & $h^{-1} \sum_{i=1}^{h} I\left\{\hat{R}_{n_{i}}-d \leq R \leq \hat{R}_{n_{i}}+d\right\}$ & Simulated value of coverage probability of $R$ \\
\hline \multirow{8}{*}{ Purely- sequential (Tables 7-10) } & $E\left[N_{d}\right]^{*}$ & $(4.3)$ & Approximation of the average stopping time \\
\hline & $\widehat{E}\left[N_{d}\right]^{*}$ & $h^{-1} \sum_{i=1}^{h} n_{i}$ & Simulated value of $E\left[N_{d}\right]^{*}$ \\
\hline & $E\left[\widehat{R}_{N_{d}}\right]^{*}$ & $(4.4)$ & Approximation of the expectation of $\widehat{R}_{N_{d}}$ \\
\hline & $\widehat{E}\left[\widehat{R}_{N_{d}}\right]^{*}$ & $h^{-1} \sum_{i=1}^{h} \hat{R}_{n_{i}}$ & Simulated value of $E\left[\widehat{R}_{N_{d}}\right]^{*}$ \\
\hline & $\operatorname{MSE}\left[\widehat{R}_{N_{d}}\right]^{*}$ & $(4.6)$ & Approximation of $M S E\left[\hat{R}_{N_{d}}\right]$ according to $R$ \\
\hline & $\widehat{M S E}^{*}$ & $h^{-1} \sum_{i=1}^{h}\left(\hat{R}_{n_{i}}-R\right)^{2}$ & Simulated value of $M S E\left[\hat{R}_{N_{d}}\right]^{*}$ according to $R$ \\
\hline & $C P^{*}$ & $(4.7)$ & Approximation of coverage probability of $R$ \\
\hline & $\widehat{C P}^{*}$ & $h^{-1} \sum_{i=1}^{h} I\left\{\hat{R}_{n_{i}}-d \leq R \leq \hat{R}_{n_{i}}+d\right\}$ & Simulated value of $C P^{*}$ \\
\hline
\end{tabular}

As Tables 3-10 show, the accuracy, precision, and coverage probability increase with $m$. The coverage probability is below the target for some cases, so we need to find the initial sample size $m$ in such a way that the coverage probability gets at least $1-\alpha$. We considered it only for the two-stage sequential procedure because we have found an exact distribution only for this case. Table 11, indicates the characteristic of the two-stage procedure and the smallest $m$ such that the coverage probability be at least $1-\alpha$. In Table 11, oversampling by the two-stage procedure is obvious. Some of the main results of the simulations are listed as follows:

1. The results derived from both of the proposed sequential procedures are almost 
the same.

2. For both proposed sequential procedures, the results of simulations and exact computations (approximations) are extremely coincide with each other, confirming the theorem's accuracy mentioned in the previous sections.

3. Given a fixed value of $m$, as $d$ decreases, $E\left[N_{m}\right]$ and $E\left[\hat{R}_{N_{m}}\right]$ increase with $E\left[\hat{R}_{N_{m}}\right]$ gets closer to $R$. Similarly, $E\left[N_{d}\right]^{*}$ and $E\left[\hat{R}_{N_{d}}\right]^{*}$ increase, with $E\left[\hat{R}_{N_{d}}\right]^{*}$ nearing to $R$.

4. Given either a fixed or decreasing value of $d$, as the initial sample size, $m$ increases, $C P\left(C P^{*}\right)$ increases, and nears its nominal value to $1-\alpha$.

5. Comparing the results in Tables 3-6 (two-stage procedure) and Tables 7-10 ( purely sequential procedure) we realize that, in the cases examined, the expected sample size in the two-stage procedure is generally larger than $n^{*}$, whereas in the sequential procedure it is generally smaller than $n^{*}$. The bias of the estimators of $R$ is negative in both procedures and is almost the same magnitude. Similar results is found about the MSE of the estimators. The coverage probabilities in both procedures are generally below the prescribed $1-\alpha$ value. These values approach the prescribed values, as $m$ getting larger. The coverage probability in the sequential procedure is more than the coverage probability in the two-stage procedure. In all cases, the purely sequential procedure is more efficient.

Although we approximate the exact distribution of $N_{d}$, one may notice that purely sequential strategy outperforms the two-stage sequential estimation strategy. Indeed, both procedures are fully expected to perform very well, but the two-stage sequential procedure is logistically more straightforward to be implemented than a purely sequential estimation one. Based on the simulation results, we can conclude that the proposed sequential procedures are working quite well, and they can be used quite effectively for data analysis purposes. 
Table 3: Characteristics and simulated values of two-stage procedure with $\alpha=0.05$, $\theta_{1}=1, \theta_{2}=2, m=5,10,20$ and $R=0.6666$.

\begin{tabular}{|c|c|c|c|c|c|c|}
\hline \multirow{2}{*}{$m$} & $n^{*}$ & 20 & 100 & 200 & 500 & 1000 \\
\hline & $d$ & 0.1377 & 0.0616 & 0.0436 & 0.0275 & 0.0195 \\
\hline \multirow{8}{*}{5} & $E\left[N_{m}\right]$ & 18.6806 & 91.0953 & 181.3078 & 454.9795 & 904.3905 \\
\hline & $\widehat{E}\left[N_{m}\right]$ & 18.6808 & 91.0525 & 181.2331 & 455.003 & 904.0718 \\
\hline & $E\left[\widehat{R}_{N_{m}}\right]$ & 0.6620 & 0.6656 & 0.6661 & 0.6665 & 0.6666 \\
\hline & $\widehat{E}\left[\widehat{R}_{N_{m}}\right]$ & 0.6622 & 0.6656 & 0.6661 & 0.6664 & 0.6665 \\
\hline & $\operatorname{MSE}\left[\widehat{R}_{N_{m}}\right]$ & $6.300 \mathrm{E}-03$ & $1.400 \mathrm{E}-03$ & 7.043E-04 & $2.820 \mathrm{E}-04$ & $1.421 \mathrm{E}-04$ \\
\hline & $\widehat{M S E}$ & $6.400 \mathrm{E}-03$ & $1.400 \mathrm{E}-03$ & 7.064E-04 & $2.819 \mathrm{E}-04$ & $1.441 \mathrm{E}-04$ \\
\hline & $C P$ & 0.9215 & 0.9130 & 0.9122 & 0.9117 & 0.9116 \\
\hline & $\widehat{C P}$ & 0.9213 & 0.9128 & 0.9119 & 0.9114 & 0.9118 \\
\hline \multirow{8}{*}{10} & $E\left[N_{m}\right]$ & 19.6836 & 95.8195 & 190.7647 & 478.7536 & 951.6705 \\
\hline & $\widehat{E}\left[N_{m}\right]$ & 19.6755 & 95.8336 & 190.7202 & 478.8408 & 951.5641 \\
\hline & $E\left[\widehat{R}_{N_{m}}\right]$ & 0.6627 & 0.6658 & 0.6662 & 0.6665 & 0.6666 \\
\hline & $\widehat{E}\left[\widehat{R}_{N_{m}}\right]$ & 0.6628 & 0.6658 & 0.6662 & 0.6665 & 0.6666 \\
\hline & $M S E\left[\hat{R}_{N_{m}}\right]$ & $5.400 \mathrm{E}-03$ & $1.100 \mathrm{E}-03$ & $5.748 \mathrm{E}-04$ & 2.292E-04 & $1.153 \mathrm{E}-04$ \\
\hline & $\widehat{M S E}$ & $5.400 \mathrm{E}-03$ & $1.100 \mathrm{E}-03$ & $5.700 \mathrm{E}-04$ & $2.289 \mathrm{E}-04$ & 1.149E-04 \\
\hline & $C P$ & 0.9401 & 0.9327 & 0.9320 & 0.9316 & 0.9315 \\
\hline & $\widehat{C P}$ & 0.9402 & 0.9324 & 0.9323 & 0.9314 & 0.9314 \\
\hline \multirow{8}{*}{20} & $E\left[N_{m}\right]$ & 21.5975 & 98.193 & 195.5053 & 490.6745 & 975.3743 \\
\hline & $\widehat{E}\left[N_{m}\right]$ & 21.6000 & 98.1677 & 195.5746 & 490.7444 & 975.5926 \\
\hline & $E\left[\widehat{R}_{N_{m}}\right]$ & 0.6633 & 0.6659 & 0.6663 & 0.6665 & 0.6666 \\
\hline & $\widehat{E}\left[\widehat{R}_{N_{m}}\right]$ & 0.6633 & 0.6659 & 0.6663 & 0.6665 & 0.6666 \\
\hline & $\operatorname{MSE}\left[\widehat{\widehat{R}}_{N_{m}}\right]$ & $4.600 \mathrm{E}-03$ & 1.100E-03 & $5.299 \mathrm{E}-04$ & 2.112E-04 & 1.062E-04 \\
\hline & $\widehat{M S E}$ & $4.600 \mathrm{E}-03$ & $1.100 \mathrm{E}-03$ & 5.316E-04 & $2.096 \mathrm{E}-04$ & $1.063 \mathrm{E}-04$ \\
\hline & $C P$ & 0.9586 & 0.9420 & 0.9415 & 0.9412 & 0.9410 \\
\hline & $\widehat{C P}$ & 0.9587 & 0.9422 & 0.9417 & 0.9415 & 0.9408 \\
\hline
\end{tabular}


Table 4: Characteristics and simulated values of two-stage procedure with $\alpha=0.05$, $\theta_{1}=1, \theta_{2}=3, m=5,10,20$ and $R=0.7500$.

\begin{tabular}{|c|c|c|c|c|c|c|}
\hline \multirow{2}{*}{$m$} & $n^{*}$ & 20 & 100 & 200 & 500 & 100 \\
\hline & $d$ & 0.1162 & 0.0520 & 0.0367 & 0.0232 & 0.0164 \\
\hline \multirow{8}{*}{5} & $E\left[N_{m}\right]$ & 20.6674 & 100.9704 & 201.4414 & 502.8482 & 1005.1994 \\
\hline & $\widehat{E}\left[N_{m}\right]$ & 20.6543 & 101.1541 & 201.8539 & 502.0154 & 1006.0814 \\
\hline & $E\left[\widehat{R}_{N_{m}}\right]$ & 0.7438 & 0.7486 & 0.7493 & 0.7497 & 0.7499 \\
\hline & $\widehat{E}\left[\widehat{R}_{N_{m}}\right]$ & 0.7438 & 0.7486 & 0.7492 & 0.7496 & 0.7498 \\
\hline & $\operatorname{MSE}\left[\widehat{R}_{N_{m}}\right]$ & 4.92E-03 & 1.10E-03 & 5.58E-04 & 2.24E-04 & $1.12 \mathrm{E}-04$ \\
\hline & $\widehat{M S E}$ & 4.94E-03 & $1.08 \mathrm{E}-03$ & 5.53E-04 & $2.26 \mathrm{E}-04$ & $1.13 \mathrm{E}-04$ \\
\hline & $C P$ & 0.9150 & 0.9051 & 0.9044 & 0.9040 & 0.9038 \\
\hline & $\widehat{C P}$ & 0.9157 & 0.9062 & 0.9051 & 0.9036 & 0.9047 \\
\hline \multirow{8}{*}{10} & $E\left[N_{m}\right]$ & 20.9463 & 101.6958 & 202.8921 & 506.4787 & 1012.4584 \\
\hline & $\widehat{E}\left[N_{m}\right]$ & 20.9310 & 101.5722 & 202.8118 & 506.4290 & 1013.5033 \\
\hline & $E\left[\widehat{R}_{N_{m}}\right]$ & 0.7449 & 0.7489 & 0.7494 & 0.7498 & 0.7499 \\
\hline & $\widehat{E}\left[\widehat{R}_{N_{m}}\right]$ & 0.7451 & 0.7488 & 0.7494 & 0.7497 & 0.7498 \\
\hline & $\operatorname{MSE}\left[\widehat{R}_{N_{m}}\right]$ & $4.01 \mathrm{E}-03$ & 8.59E-04 & $4.30 \mathrm{E}-04$ & $1.72 \mathrm{E}-04$ & 8.59E-05 \\
\hline & $\widehat{M S E}$ & 4.04E-03 & 8.64E-04 & 4.34E-04 & $1.71 \mathrm{E}-04$ & 8.60E-05 \\
\hline & $C P$ & 0.9361 & 0.9268 & 0.9264 & 0.9261 & 0.9260 \\
\hline & $\widehat{C P}$ & 0.9354 & 0.9269 & 0.9248 & 0.9263 & 0.9261 \\
\hline \multirow{8}{*}{20} & $E\left[N_{m}\right]$ & 22.7565 & 101.4015 & 202.3025 & 505.0060 & 1009.5122 \\
\hline & $\widehat{E}\left[N_{m}\right]$ & 22.7634 & 101.4896 & 202.2260 & 505.0895 & 1008.7894 \\
\hline & $E\left[\widehat{R}_{N_{m}}\right]$ & 0.7458 & 0.7490 & 0.7495 & 0.7498 & 0.7499 \\
\hline & $\widehat{E}\left[\widehat{R}_{N_{m}}\right]$ & 0.7457 & 0.7490 & 0.7495 & 0.7498 & 0.7499 \\
\hline & $\operatorname{MSE}\left[\widehat{R}_{N_{m}}\right]$ & $3.25 \mathrm{E}-03$ & 7.73E-04 & $3.86 \mathrm{E}-04$ & $1.54 \mathrm{E}-04$ & 7.72E-05 \\
\hline & $\widehat{M S E}$ & $3.24 \mathrm{E}-03$ & 7.75E-04 & 3.87E-04 & $1.54 \mathrm{E}-04$ & 7.77E-05 \\
\hline & $C P$ & 0.9587 & 0.9384 & 0.9381 & 0.9379 & 0.9378 \\
\hline & $\widehat{C P}$ & 0.9594 & 0.9378 & 0.9374 & 0.9390 & 0.9365 \\
\hline
\end{tabular}


Table 5: Characteristics and simulated values of two-stage procedure with $\alpha=0.05$, $\theta_{1}=1, \theta_{2}=5, m=5,10,20$ and $R=0.8333$.

\begin{tabular}{|c|c|c|c|c|c|c|}
\hline \multirow{2}{*}{$m$} & $n^{*}$ & 20 & 100 & 200 & 500 & 1000 \\
\hline & $d$ & 0.0861 & 0.0385 & 0.0272 & 0.0172 & 0.0122 \\
\hline \multirow{8}{*}{5} & $E\left[N_{m}\right]$ & 24.2817 & 118.8283 & 237.1490 & 592.1213 & 1183.7429 \\
\hline & $\widehat{E}\left[N_{m}\right]$ & 24.2631 & 118.9932 & 236.8460 & 591.1848 & 1176.5900 \\
\hline & $E\left[\widehat{R}_{N_{m}}\right]$ & 0.8269 & 0.8317 & 0.8325 & 0.8330 & 0.8332 \\
\hline & $\widehat{E}\left[\widehat{R}_{N_{m}}\right]$ & 0.8268 & 0.8317 & 0.8326 & 0.8330 & 0.8332 \\
\hline & $M S E\left[\widehat{R}_{N_{m}}\right]$ & 3.09E-03 & $7.08 \mathrm{E}-04$ & $3.56 \mathrm{E}-04$ & $1.42 \mathrm{E}-04$ & 7.08E-05 \\
\hline & $\widehat{M S E}$ & 3.10E-03 & 7.10E-04 & 3.58E-04 & $1.43 \mathrm{E}-04$ & 7.27E-05 \\
\hline & $C P$ & 0.9074 & 0.8945 & 0.8936 & 0.8932 & 0.8930 \\
\hline & $\overline{C P}$ & 0.9069 & 0.8947 & 0.8936 & 0.8937 & 0.8917 \\
\hline \multirow{8}{*}{10} & $E\left[N_{m}\right]$ & 22.9999 & 111.2373 & 221.9707 & 554.1766 & 1107.8533 \\
\hline & $\widehat{E}\left[N_{m}\right]$ & 22.9813 & 111.3915 & 221.7133 & 553.5728 & 1107.2376 \\
\hline & $E\left[\widehat{R}_{N_{m}}\right]$ & 0.8282 & 0.8321 & 0.8327 & 0.8331 & 0.8332 \\
\hline & $\widehat{E}\left[\widehat{R}_{N_{m}}\right]$ & 0.8281 & 0.8322 & 0.8328 & 0.8331 & 0.8333 \\
\hline & $M S E\left[\widehat{R}_{N_{m}}\right]$ & $2.34 \mathrm{E}-03$ & 5.10E-04 & 2.53E-04 & $1.01 \mathrm{E}-04$ & 5.02E-05 \\
\hline & $\widehat{M S E}$ & 2.35E-03 & $5.02 \mathrm{E}-04$ & 2.53E-04 & $1.01 \mathrm{E}-04$ & 5.00E-05 \\
\hline & $C P$ & 0.9317 & 0.9194 & 0.9190 & 0.9188 & 0.9187 \\
\hline & $\widehat{C P}$ & 0.9317 & 0.9207 & 0.9190 & 0.9186 & 0.9186 \\
\hline \multirow{8}{*}{20} & $E\left[N_{m}\right]$ & 24.1967 & 106.2557 & 212.0079 & 529.2696 & 1058.0393 \\
\hline & $\widehat{E}\left[N_{m}\right]$ & 24.2183 & 106.2909 & 212.1401 & 530.3853 & 1059.8462 \\
\hline & $E\left[\widehat{R}_{N_{m}}\right]$ & 0.8293 & 0.8323 & 0.8328 & 0.8331 & 0.8332 \\
\hline & $\widehat{E}\left[\widehat{R}_{N_{m}}\right]$ & 0.8294 & 0.8323 & 0.8328 & 0.8331 & 0.8332 \\
\hline & $M S E\left[\widehat{R}_{N_{m}}\right]$ & $1.79 \mathrm{E}-03$ & 4.42E-04 & 2.20E-04 & 8.75E-05 & 4.37E-05 \\
\hline & $\widehat{M S E}$ & 1.79E-03 & 4.41E-04 & 2.19E-04 & 8.71E-05 & $4.40 \mathrm{E}-05$ \\
\hline & $C P$ & 0.9572 & 0.9337 & 0.9335 & 0.9335 & 0.9335 \\
\hline & $\widehat{C P}$ & 0.9572 & 0.9331 & 0.9336 & 0.9335 & 0.9322 \\
\hline
\end{tabular}


108 A. khalifeh et al.

Table 6: Characteristics and simulated values of two-stage procedure with $\alpha=0.05$, $\theta_{1}=1, \theta_{2}=7, m=5,10,20$ and $R=0.875$.

\begin{tabular}{|c|c|c|c|c|c|c|}
\hline \multirow{2}{*}{$m$} & $n^{*}$ & 20 & 100 & 200 & 500 & 1000 \\
\hline & $d$ & 0.0678 & 0.0303 & 0.0214 & 0.0136 & 0.0096 \\
\hline \multirow{8}{*}{5} & $E\left[N_{m}\right]$ & 27.1543 & 133.0503 & 265.5876 & 663.2155 & 1325.9306 \\
\hline & $\widehat{E}\left[N_{m}\right]$ & 27.1849 & 132.9368 & 266.0099 & 663.1108 & 1329.4560 \\
\hline & $E\left[\widehat{R}_{N_{m}}\right]$ & 0.8690 & 0.8735 & 0.874217 & 0.8747 & 0.8748 \\
\hline & $\widehat{E}\left[\widehat{R}_{N_{m}}\right]$ & 0.8689 & 0.8734 & 0.874237 & 0.8747 & 0.8748 \\
\hline & $\operatorname{MSE}\left[\widehat{R}_{N_{m}}\right]$ & 2.10E-03 & 4.84E-04 & 2.42E-04 & $9.60 \mathrm{E}-05$ & 4.76E-05 \\
\hline & $\widehat{M S E}$ & 2.09E-03 & $4.84 \mathrm{E}-04$ & $2.40 \mathrm{E}-04$ & $9.56 \mathrm{E}-05$ & 4.84E-05 \\
\hline & $C P$ & 0.9038 & 0.8887 & 0.887705 & 0.8872 & 0.8871 \\
\hline & $\widehat{C P}$ & 0.9042 & 0.8882 & 0.88737 & 0.8869 & 0.8865 \\
\hline \multirow{8}{*}{10} & $E\left[N_{m}\right]$ & 24.4308 & 117.9825 & 235.4582 & 587.8949 & 1175.2899 \\
\hline & $\widehat{E}\left[N_{m}\right]$ & 24.3310 & 118.0597 & 235.364 & 588.6521 & 1177.0300 \\
\hline & $E\left[\widehat{R}_{N_{m}}\right]$ & 0.8704 & 0.8739 & 0.874449 & 0.8748 & 0.8749 \\
\hline & $\widehat{E}\left[\widehat{R}_{N_{m}}\right]$ & 0.8705 & 0.8738 & 0.874447 & 0.8748 & 0.8749 \\
\hline & $\operatorname{MSE}\left[\widehat{R}_{N_{m}}\right]$ & $1.51 \mathrm{E}-03$ & 3.32E-04 & $1.64 \mathrm{E}-04$ & $6.50 \mathrm{E}-05$ & $3.24 \mathrm{E}-05$ \\
\hline & $\widehat{M S E}$ & $1.49 \mathrm{E}-03$ & 3.34E-04 & $1.65 \mathrm{E}-04$ & 6.57E-05 & 3.27E-05 \\
\hline & $C P$ & 0.9296 & 0.9153 & 0.914875 & 0.9147 & 0.9146 \\
\hline & $\widehat{C P}$ & 0.9300 & 0.9146 & 0.91502 & 0.9131 & 0.9148 \\
\hline \multirow{8}{*}{20} & $E\left[N_{m}\right]$ & 25.0496 & 109.4479 & 218.3886 & 545.2214 & 1089.9429 \\
\hline & $\widehat{E}\left[N_{m}\right]$ & 25.0325 & 109.3863 & 218.7623 & 546.4983 & 1093.5911 \\
\hline & $E\left[\widehat{R}_{N_{m}}\right]$ & 0.8714 & 0.8741 & 0.874528 & 0.8748 & 0.8749 \\
\hline & $\widehat{E}\left[\widehat{R}_{N_{m}}\right]$ & 0.8715 & 0.8740 & 0.874464 & 0.8748 & 0.8749 \\
\hline & $\operatorname{MSE}\left[\widehat{R}_{N_{m}}\right]$ & $1.12 \mathrm{E}-03$ & 2.81E-04 & $1.39 \mathrm{E}-04$ & $5.54 \mathrm{E}-05$ & $2.76 \mathrm{E}-05$ \\
\hline & $\widehat{M S E}$ & $1.12 \mathrm{E}-03$ & 2.83E-04 & $1.39 \mathrm{E}-04$ & 5.49E-05 & 2.73E-05 \\
\hline & $C P$ & 0.9561 & 0.9310 & 0.930961 & 0.9310 & 0.9310 \\
\hline & $\widehat{C P}$ & 0.9574 & 0.9308 & 0.93061 & 0.9324 & 0.9319 \\
\hline
\end{tabular}


Table 7: Characteristics and simulated values of purely sequential procedure with $\alpha=0.05, \theta_{1}=1, \theta_{2}=2, m=5,10,20$ and $R=0.6666$.

\begin{tabular}{|c|c|c|c|c|c|c|}
\hline \multirow[b]{2}{*}{$m$} & $n^{*}$ & 20 & 100 & 200 & 500 & 1000 \\
\hline & $d$ & 0.1377 & 0.0616 & 0.0436 & 0.0275 & 0.0195 \\
\hline \multirow{8}{*}{5} & $E\left[N_{d}\right]^{*}$ & 18.9280 & 99.2708 & 199.0051 & 501.1108 & 997.0738 \\
\hline & $\widehat{E}\left[N_{d}\right]^{*}$ & 18.9148 & 99.2436 & 199.1469 & 501.6570 & 997.9636 \\
\hline & $E\left[\widehat{R}_{N_{d}}\right]^{*}$ & 0.6623 & 0.6659 & 0.6663 & 0.6665 & 0.6666 \\
\hline & $\widehat{E}\left[\widehat{R}_{N_{d}}\right]_{-}^{*}$ & 0.6622 & 0.6659 & 0.6663 & 0.6665 & 0.6666 \\
\hline & $\operatorname{MSE}\left[\widehat{R}_{N_{d}}\right]^{*}$ & $6.000 \mathrm{E}-03$ & $1.000 \mathrm{E}-03$ & $5.033 \mathrm{E}-04$ & $1.975 \mathrm{E}-04$ & 9.912E-05 \\
\hline & $\widehat{M S E}^{*}$ & 6.100E-03 & $1.000 \mathrm{E}-03$ & $5.035 \mathrm{E}-04$ & $1.949 \mathrm{E}-04$ & 9.733E-05 \\
\hline & $C P^{*}$ & 0.9281 & 0.9470 & 0.9493 & 0.9496 & 0.9499 \\
\hline & $\widehat{C P^{*}}$ & 0.9286 & 0.9474 & 0.9491 & 0.9498 & 0.9498 \\
\hline \multirow{8}{*}{10} & $E\left[N_{N_{d}}\right]^{*}$ & 19.4449 & 99.3574 & 199.2510 & 500.8986 & 997.0674 \\
\hline & $\widehat{E}\left[N_{N_{d}}\right]^{*}$ & 19.4396 & 99.2483 & 199.1443 & 501.1976 & 997.2442 \\
\hline & $E\left[\widehat{R}_{N_{d}}\right]^{*}$ & 0.6627 & 0.6659 & 0.6663 & 0.6665 & 0.6666 \\
\hline & $\widehat{E}\left[\widehat{R}_{N_{d}}\right]_{-}^{*}$ & 0.6627 & 0.6659 & 0.6663 & 0.6665 & 0.6666 \\
\hline & $\operatorname{MSE}\left[\widehat{R}_{N_{d}}\right]^{*}$ & $5.400 \mathrm{E}-03$ & $1.000 \mathrm{E}-03$ & 4.987E-04 & $1.975 \mathrm{E}-04$ & 9.907E-05 \\
\hline & $\widehat{M S E}^{*}$ & 5.400E-03 & $1.000 \mathrm{E}-03$ & $5.006 \mathrm{E}-04$ & $1.957 \mathrm{E}-04$ & 9.982E-05 \\
\hline & $C P^{*}$ & 0.9408 & 0.9480 & 0.9493 & 0.9497 & 0.9499 \\
\hline & $\widehat{C P^{*}}$ & 0.9400 & 0.9477 & 0.9492 & 0.9496 & 0.9498 \\
\hline \multirow{8}{*}{20} & $E\left[N_{N_{d}}\right]^{*}$ & 21.5103 & 99.3548 & 199.2791 & 501.4209 & 997.3726 \\
\hline & $\widehat{E}\left[N_{N_{d}}\right]^{*}$ & 21.5255 & 99.2791 & 199.1347 & 501.3926 & 997.3315 \\
\hline & $E\left[\widehat{R}_{N_{d}}\right]^{*}$ & 0.6633 & 0.6659 & 0.6663 & 0.6665 & 0.6666 \\
\hline & $\widehat{E}\left[\widehat{R}_{N_{d}}\right]^{*}$ & 0.6633 & 0.6659 & 0.6663 & 0.6665 & 0.6666 \\
\hline & $\operatorname{MSE}\left[\widehat{R}_{N_{d}}\right]^{*}$ & 4.600E-03 & $1.000 \mathrm{E}-03$ & $4.986 \mathrm{E}-04$ & $1.974 \mathrm{E}-04$ & 9.912E-05 \\
\hline & $\widehat{M S E}^{*}$ & 4.700E-03 & $1.000 \mathrm{E}-03$ & 4.872E-04 & $1.934 \mathrm{E}-04$ & $9.568 \mathrm{E}-05$ \\
\hline & $C P^{*}$ & 0.9588 & 0.9481 & 0.9493 & 0.9499 & 0.9499 \\
\hline & $\widehat{C P^{*}}$ & 0.9583 & 0.9481 & 0.9493 & 0.9498 & 0.9499 \\
\hline
\end{tabular}


Table 8: Characteristics and simulated values of purely sequential procedure with $\alpha=0.05, \theta_{1}=1, \theta_{2}=3, m=5,10,20$ and $R=0.7500$.

\begin{tabular}{|c|c|c|c|c|c|c|}
\hline \multirow{2}{*}{$m$} & $n^{*}$ & 20 & 100 & 200 & 500 & 1000 \\
\hline & $d$ & 0.1162 & 0.0520 & 0.0367 & 0.0232 & 0.0164 \\
\hline \multirow{8}{*}{5} & $E\left[N_{d}\right]^{*}$ & 18.6937 & 98.9637 & 198.9288 & 498.8600 & 998.2622 \\
\hline & $\widehat{E}\left[N_{d}\right]^{*}$ & 18.8218 & 98.9522 & 199.0617 & 499.5763 & 999.2656 \\
\hline & $E\left[\widehat{R}_{N_{d}}\right]^{*}$ & 0.7437 & 0.7490 & 0.7495 & 0.7498 & 0.749906 \\
\hline & $\widehat{E}\left[\widehat{R}_{N_{d}}\right]^{*}$ & 0.7446 & 0.7492 & 0.7495 & 0.7495 & 0.749953 \\
\hline & $\operatorname{MSE}\left[\widehat{R}_{N_{d}}\right]^{*}$ & $5.00 \mathrm{E}-03$ & 7.66E-04 & 3.63E-04 & $1.42 \mathrm{E}-04$ & 7.06E-05 \\
\hline & $\widehat{M S E}^{*}$ & $4.81 \mathrm{E}-03$ & 7.53E-04 & $3.62 \mathrm{E}-04$ & $1.42 \mathrm{E}-04$ & 6.82E-05 \\
\hline & $C P^{*}$ & 0.9135 & 0.9439 & 0.9475 & 0.9491 & 0.949495 \\
\hline & $\widehat{C P}^{*}$ & 0.9183 & 0.9434 & 0.9457 & 0.9496 & 0.9534 \\
\hline \multirow{8}{*}{10} & $E\left[N_{d}\right]^{*}$ & 19.6192 & 99.1603 & 199.3606 & 499.1454 & 999.3403 \\
\hline & $\widehat{E}\left[N_{d}\right]^{*}$ & 19.5277 & 99.0741 & 199.5256 & 499.0761 & 999.4349 \\
\hline & $E\left[\widehat{R}_{N_{d}}\right]^{*}$ & 0.7447 & 0.7490 & 0.7495 & 0.7498 & 0.749906 \\
\hline & $\widehat{E}\left[\widehat{R}_{N_{d}}\right]^{*}$ & 0.7447 & 0.7491 & 0.7494 & 0.7498 & 0.74976 \\
\hline & $\operatorname{MSE}\left[\widehat{R}_{N_{d}}\right]^{*}$ & 4.13E-03 & 7.37E-04 & $3.58 \mathrm{E}-04$ & $1.42 \mathrm{E}-04$ & 7.05E-05 \\
\hline & $\widehat{M S E}^{*}$ & $4.14 \mathrm{E}-03$ & 7.51E-04 & $3.58 \mathrm{E}-04$ & $1.40 \mathrm{E}-04$ & $7.08 \mathrm{E}-05$ \\
\hline & $C P^{*}$ & 0.9331 & 0.9450 & 0.9480 & 0.9492 & 0.949624 \\
\hline & $\widehat{C P}^{*}$ & 0.9358 & 0.9430 & 0.9489 & 0.9513 & 0.9487 \\
\hline \multirow{8}{*}{20} & $E\left[N_{d}\right]^{*}$ & 22.3833 & 99.1211 & 199.1688 & 499.3151 & 999.6274 \\
\hline & $\widehat{E}\left[N_{d}\right]^{*}$ & 22.3847 & 99.3918 & 199.3486 & 499.2030 & 999.8737 \\
\hline & $E\left[\widehat{R}_{N_{d}}\right]^{*}$ & 0.7458 & 0.7490 & 0.7495 & 0.7498 & 0.749906 \\
\hline & $\widehat{E}\left[\widehat{R}_{N_{d}}\right]^{*}$ & 0.7465 & 0.7491 & 0.7495 & 0.7497 & 0.749952 \\
\hline & $\operatorname{MSE}\left[\widehat{R}_{N_{d}}\right]^{*}$ & $3.28 \mathrm{E}-03$ & 7.34E-04 & $3.58 \mathrm{E}-04$ & $1.42 \mathrm{E}-04$ & 7.05E-05 \\
\hline & $\widehat{M S E}^{*}$ & $3.24 \mathrm{E}-03$ & 7.53E-04 & $3.56 \mathrm{E}-04$ & $1.41 \mathrm{E}-04$ & 7.14E-05 \\
\hline & $C P^{*}$ & 0.9577 & 0.9452 & 0.9479 & 0.9492 & 0.9496 \\
\hline & $\widehat{C P}^{*}$ & 0.9607 & 0.9421 & 0.9501 & 0.9509 & 0.9477 \\
\hline
\end{tabular}


Table 9: Characteristics and simulated values of purely sequential procedure with $\alpha=0.05, \theta_{1}=1, \theta_{2}=5, m=5,10,20$ and $R=0.8333$.

\begin{tabular}{|c|c|c|c|c|c|c|}
\hline \multirow{2}{*}{$m$} & $n^{*}$ & 20 & 100 & 200 & 500 & 1000 \\
\hline & $d$ & 0.0861 & 0.0385 & 0.0272 & 0.0172 & 0.0122 \\
\hline \multirow{8}{*}{5} & $E\left[N_{d}\right]^{*}$ & 18.6361 & 98.0376 & 198.2179 & 498.7504 & 999.1778 \\
\hline & $\widehat{E}\left[N_{d}\right]^{*}$ & 18.5870 & 98.2458 & 198.3566 & 498.7384 & 999.5550 \\
\hline & $E\left[\widehat{R}_{N_{d}}\right]^{*}$ & 0.8265 & 0.8322 & 0.8328 & 0.8331 & 0.8332 \\
\hline & $\widehat{E}\left[\widehat{R}_{N_{d}}\right]^{*}$ & 0.8268 & 0.8319 & 0.8328 & 0.8332 & 0.8333 \\
\hline & $\operatorname{MSE}\left[\widehat{R}_{N_{d}}\right]^{*}$ & $3.26 \mathrm{E}-03$ & 4.91E-04 & 2.15E-04 & 7.91E-05 & 3.88E-05 \\
\hline & $\widehat{M S E}^{*}$ & $3.29 \mathrm{E}-03$ & 4.67E-04 & 2.03E-04 & 7.78E-05 & 3.84E-05 \\
\hline & $C P^{*}$ & 0.9009 & 0.9360 & 0.9447 & 0.9484 & 0.9493 \\
\hline & $\widehat{C P}^{*}$ & 0.9034 & 0.9350 & 0.9457 & 0.9480 & 0.9500 \\
\hline \multirow{8}{*}{10} & $E\left[N_{d}\right]^{*}$ & 19.6835 & 98.2149 & 198.9431 & 499.5781 & 1000.3711 \\
\hline & $\widehat{E}\left[N_{d}\right]^{*}$ & 19.9368 & 98.4462 & 199.0357 & 498.5999 & 999.3145 \\
\hline & $E\left[\widehat{R}_{N_{d}}\right]^{*}$ & 0.8278 & 0.8323 & 0.8329 & 0.8331 & 0.8332 \\
\hline & $\widehat{E}\left[\widehat{R}_{N_{d}}\right]^{*}$ & 0.8279 & 0.8322 & 0.8330 & 0.8331 & 0.8332 \\
\hline & $\operatorname{MSE}\left[\widehat{R}_{N_{d}}\right]^{*}$ & $2.51 \mathrm{E}-03$ & $4.28 \mathrm{E}-04$ & 2.00E-04 & 7.80E-05 & 3.88E-05 \\
\hline & $\widehat{M S E}^{*}$ & $2.49 \mathrm{E}-03$ & 4.21E-04 & $1.99 \mathrm{E}-04$ & 7.77E-05 & $3.96 \mathrm{E}-05$ \\
\hline & $C P^{*}$ & 0.9246 & 0.9395 & 0.9461 & 0.9487 & 0.9494 \\
\hline & $\widehat{C P}^{*}$ & 0.9255 & 0.9438 & 0.9468 & 0.9455 & 0.9458 \\
\hline \multirow{8}{*}{20} & $E\left[N_{d}\right]^{*}$ & 23.1967 & 98.7593 & 198.9704 & 498.2473 & 998.8203 \\
\hline & $\bar{E}\left[N_{d}\right]^{*}$ & 23.1966 & 98.6641 & 198.5786 & 499.0432 & 1000.2725 \\
\hline & $E\left[\widehat{R}_{N_{d}}\right]^{*}$ & 0.8292 & 0.8323 & 0.8329 & 0.8331 & 0.8332 \\
\hline & $\widehat{E}\left[\widehat{R}_{N_{d}}\right]^{*}$ & 0.8285 & 0.8322 & 0.8327 & 0.8331 & 0.8332 \\
\hline & $\operatorname{MSE}\left[\widehat{R}_{N_{d}}\right]^{*}$ & $1.83 \mathrm{E}-03$ & 4.17E-04 & $1.99 \mathrm{E}-04$ & 7.83E-05 & 3.88E-05 \\
\hline & $\widehat{M S E}^{*}$ & $1.87 \mathrm{E}-03$ & 4.28E-04 & 2.02E-04 & 7.74E-05 & 3.88E-05 \\
\hline & $C P^{*}$ & 0.9555 & 0.9414 & 0.9462 & 0.9484 & 0.9493 \\
\hline & $\widehat{C P}^{*}$ & 0.9523 & 0.9392 & 0.9447 & 0.9519 & 0.9484 \\
\hline
\end{tabular}


Table 10: Characteristics and simulated values of purely sequential procedure with $\alpha=0.05, \theta_{1}=1, \theta_{2}=7, m=5,10,20$ and $R=0.875$.

\begin{tabular}{|c|c|c|c|c|c|c|}
\hline \multirow{2}{*}{$m$} & $n^{*}$ & 20 & 100 & 200 & 500 & 1000 \\
\hline & $d$ & 0.0678 & 0.0303 & 0.0214 & 0.0136 & 0.0096 \\
\hline \multirow{8}{*}{5} & $E\left[N_{d}\right]^{*}$ & 18.5717 & 97.6680 & 198.3381 & 498.8221 & 999.3101 \\
\hline & $\widehat{E}\left[N_{d}\right]^{*}$ & 18.5204 & 96.8969 & 198.2913 & 499.5355 & 998.3575 \\
\hline & $E\left[\widehat{R}_{N_{d}}\right]^{*}$ & 0.8685 & 0.8739 & 0.8745 & 0.8748 & 0.8749 \\
\hline & $\widehat{E}\left[\widehat{R}_{N_{d}}\right]^{*}$ & 0.8688 & 0.8743 & 0.8746 & 0.8749 & 0.8749 \\
\hline & $\operatorname{MSE}\left[\widehat{R}_{N_{d}}\right]^{*}$ & 2.27E-03 & $3.29 \mathrm{E}-04$ & $1.38 \mathrm{E}-04$ & 5.03E-05 & $2.54 \mathrm{E}-05$ \\
\hline & $\widehat{M S E}^{*}$ & $2.23 \mathrm{E}-03$ & $3.59 \mathrm{E}-04$ & 1.37E-04 & 4.93E-05 & 2.42E-05 \\
\hline & $C P^{*}$ & 0.8937 & 0.9320 & 0.9435 & 0.9479 & 0.9490 \\
\hline & $\widehat{C P}^{*}$ & 0.8954 & 0.9305 & 0.9405 & 0.9469 & 0.9451 \\
\hline \multirow{8}{*}{10} & $E\left[N_{d}\right]^{*}$ & 19.7899 & 98.2148 & 198.4795 & 498.5058 & 998.1902 \\
\hline & $\widehat{E}\left[N_{d}\right]^{*}$ & 20.1036 & 98.1968 & 198.2880 & 498.6699 & 999.7197 \\
\hline & $E\left[\widehat{R}_{N_{d}}\right]^{*}$ & 0.8700 & 0.8741 & 0.8746 & 0.8748 & 0.8749 \\
\hline & $\widehat{E}\left[\widehat{R}_{N_{d}}\right]^{*}$ & 0.8707 & 0.8740 & 0.8745 & 0.8748 & 0.8749 \\
\hline & $\operatorname{MSE}\left[\widehat{R}_{N_{d}}\right]^{*}$ & $1.64 \mathrm{E}-03$ & 2.77E-04 & $1.25 \mathrm{E}-04$ & 4.87E-05 & 2.42E-05 \\
\hline & $\widehat{M S E}^{*}$ & $1.53 \mathrm{E}-03$ & $2.80 \mathrm{E}-04$ & $1.25 \mathrm{E}-04$ & 4.82E-05 & 2.43E-05 \\
\hline & $C P^{*}$ & 0.9208 & 0.9365 & 0.9450 & 0.9481 & 0.9490 \\
\hline & $\widehat{C P}^{*}$ & 0.9275 & 0.9360 & 0.9434 & 0.9489 & 0.9487 \\
\hline \multirow{8}{*}{20} & $E\left[N_{d}\right]^{*}$ & 23.6220 & 97.9493 & 199.1339 & 498.9835 & 998.5301 \\
\hline & $\widehat{E}\left[N_{d}\right]^{*}$ & 23.5805 & 98.3716 & 198.3858 & 498.8461 & 999.3820 \\
\hline & $E\left[\widehat{R}_{N_{d}}\right]^{*}$ & 0.8713 & 0.8741 & 0.8746 & 0.8748 & 0.8749 \\
\hline & $\widehat{E}\left[\widehat{R}_{N_{d}}\right]^{*}$ & 0.8715 & 0.8741 & 0.8746 & 0.8748 & 0.8750 \\
\hline & $\operatorname{MSE}\left[\widehat{R}_{N_{d}}\right]^{*}$ & $1.15 \mathrm{E}-03$ & 2.69E-04 & $1.25 \mathrm{E}-04$ & $4.86 \mathrm{E}-05$ & $2.42 \mathrm{E}-05$ \\
\hline & $\widehat{M S E}^{*}$ & $1.18 \mathrm{E}-03$ & 2.71E-04 & 1.27E-04 & $5.04 \mathrm{E}-05$ & 2.37E-05 \\
\hline & $C P^{*}$ & 0.9539 & 0.9376 & 0.9451 & 0.9482 & 0.9491 \\
\hline & $\widehat{C P}^{*}$ & 0.9524 & 0.9354 & 0.9450 & 0.9421 & 0.9513 \\
\hline
\end{tabular}


Table 11: Characteristic of the two-stage procedure and the smallest $m$ such that coverage probability be at least $95 \%$.

\begin{tabular}{|c|c|c|c|c|c|c|}
\hline & $n^{*}$ & 20 & 100 & 200 & 500 & 1000 \\
\hline \multirow{10}{*}{$\theta_{1}=1, \theta_{2}=2$} & $d$ & 0.1377 & 0.0616 & 0.0616 & 0.0275 & 0.0195 \\
\hline & $m$ & 16 & 88 & 181 & 464 & 939 \\
\hline & $E\left[N_{m}\right]$ & 20.4368 & 100.6199 & 200.6407 & 500.5397 & 1000.3440 \\
\hline & $\widehat{E}\left[N_{m}\right]$ & 20.4368 & 100.6246 & 200.6659 & 500.6219 & 1000.4066 \\
\hline & $E\left[\widehat{R}_{N_{m}}\right]$ & 0.6630 & 0.6659 & 0.6663 & 0.6665 & 0.6666 \\
\hline & $\widehat{E}\left[\widehat{R}_{N_{m}}\right]$ & 0.6631 & 0.6660 & 0.6663 & 0.6665 & 0.6666 \\
\hline & $\operatorname{MSE}\left[\widehat{R}_{N_{m}}\right]$ & 5.00E-03 & 9.89E-04 & 5.00E-04 & 2.00E-04 & $9.88 \mathrm{E}-05$ \\
\hline & $\widehat{M S E}$ & 4.90E-03 & 9.91E-04 & 5.00E-04 & 2.00E-04 & 9.90E-05 \\
\hline & $C P$ & 0.9505 & 0.9500 & 0.9500 & 0.9500 & 0.9500 \\
\hline & $\widehat{C P}$ & 0.9507 & 0.9503 & 0.9493 & 0.9496 & 0.9495 \\
\hline \multirow{10}{*}{$\theta_{1}=1, \theta_{2}=3$} & $d$ & 0.1162 & 0.0520 & 0.0367 & 0.0232 & 0.0164 \\
\hline & $m$ & 17 & 87 & 178 & 458 & 931 \\
\hline & $E\left[N_{m}\right]$ & 21.7933 & 102.1451 & 202.1986 & 502.2104 & 1002.0449 \\
\hline & $\widehat{E}\left[N_{m}\right]$ & 21.8044 & 102.1311 & 202.2000 & 502.1621 & 1001.9552 \\
\hline & $E\left[\widehat{R}_{N_{m}}\right]$ & 0.7455 & 0.7491 & 0.7495 & 0.7498 & 0.7499 \\
\hline & $\widehat{E}\left[\widehat{R}_{N_{m}}\right]$ & 0.7455 & 0.7490 & 0.7495 & 0.7498 & 0.7499 \\
\hline & $\operatorname{MSE}\left[\widehat{R}_{N_{m}}\right]$ & $3.48 \mathrm{E}-03$ & 7.03E-04 & 3.52E-04 & $1.41 \mathrm{E}-04$ & 7.03E-05 \\
\hline & $\widehat{M S E}$ & $3.48 \mathrm{E}-03$ & 7.03E-04 & 3.53E-04 & $1.40 \mathrm{E}-04$ & 7.06E-05 \\
\hline & $C P$ & 0.9518 & 0.9501 & 0.9500 & 0.9500 & 0.9500 \\
\hline & $\widehat{C P}$ & 0.9516 & 0.9491 & 0.9495 & 0.9501 & 0.9498 \\
\hline \multirow{10}{*}{$\theta_{1}=1, \theta_{2}=5$} & $d$ & 0.0861 & 0.0385 & 0.0272 & 0.0172 & 0.0122 \\
\hline & $m$ & 17 & 86 & 176 & 452 & 922 \\
\hline & $E\left[N_{m}\right]$ & 23.3406 & 104.1099 & 204.3535 & 504.4301 & 1004.4449 \\
\hline & $\widehat{E}\left[N_{m}\right]$ & 23.2954 & 104.1361 & 204.3072 & 504.3212 & 1004.7583 \\
\hline & $E\left[\widehat{R}_{N_{m}}\right]$ & 0.8290 & 0.8324 & 0.8329 & 0.8331 & 0.8332 \\
\hline & $\widehat{E}\left[\widehat{R}_{N_{m}}\right]$ & 0.8290 & 0.8325 & 0.8329 & 0.8331 & 0.8332 \\
\hline & $\operatorname{MSE}\left[\widehat{R}_{N_{m}}\right]$ & 1.93E-03 & $3.86 \mathrm{E}-04$ & 1.93E-04 & 7.72E-05 & $3.86 \mathrm{E}-05$ \\
\hline & $\widehat{M S E}$ & 1.93E-03 & 3.89E-04 & 1.93E-04 & 7.66E-05 & $3.86 \mathrm{E}-05$ \\
\hline & $C P$ & 0.9501 & 0.9500 & 0.9500 & 0.9500 & 0.9500 \\
\hline & $\widehat{C P}$ & 0.9503 & 0.9496 & 0.9503 & 0.9508 & 0.9497 \\
\hline \multirow{10}{*}{$\theta_{1}=1, \theta_{2}=7$} & $d$ & 0.0678 & 0.0303 & 0.0214 & 0.0136 & 0.0096 \\
\hline & $m$ & 17 & 86 & 175 & 450 & 917 \\
\hline & $E\left[N_{m}\right]$ & 24.4921 & 104.1099 & 205.5915 & 505.8347 & 1005.8066 \\
\hline & $\widehat{E}\left[N_{m}\right]$ & 24.5044 & 104.1459 & 205.5118 & 506.0394 & 1005.5856 \\
\hline & $E\left[\widehat{R}_{N_{m}}\right]$ & 0.8713 & 0.8324 & 0.8746 & 0.8748 & 0.8749 \\
\hline & $\widehat{E}\left[\widehat{R}_{N_{m}}\right]$ & 0.8713 & 0.8324 & 0.8746 & 0.8748 & 0.8749 \\
\hline & $\operatorname{MSE}\left[\widehat{R}_{N_{m}}\right]$ & $1.18 \mathrm{E}-03$ & $3.86 \mathrm{E}-04$ & $1.20 \mathrm{E}-04$ & 4.79E-05 & $2.40 \mathrm{E}-05$ \\
\hline & $\widehat{M S E}$ & 1.17E-03 & $3.84 \mathrm{E}-04$ & $1.21 \mathrm{E}-04$ & 4.78E-05 & 2.39E-05 \\
\hline & $C P$ & 0.9513 & 0.9500 & 0.9500 & 0.9500 & 0.9500 \\
\hline & $\widehat{C P}$ & 0.9517 & 0.9498 & 0.9490 & 0.9501 & 0.9501 \\
\hline
\end{tabular}




\section{Real Data}

In this section, the analysis of a pair of real datasets is presented for illustrative purposes. Breaking strengths of jute fiber into two different gauge lengths are shown in Table 1213. These two datasets were used by Xia et al. (2009) and presented earlier in Mirjalili et al. (2016). According to the latter, the data in Tables 12-13 have exponential distribution. Let random variables, $X$ and $Y$ be breaking strengths of jute fiber of gauge length $10 \mathrm{~mm}$ and $20 \mathrm{~mm}$, respectively. Define reliability parameter under the stress-strength model, as the probability that jute fiber of gauge length $20 \mathrm{~mm}$ endures more than that of gauge length $10 \mathrm{~mm}$.

Table 12: Dataset 1 (Breaking strength of jute fiber of gauge length $10 \mathrm{~mm}$ ).

\begin{tabular}{cccccccccc}
\hline 693.73 & 704.66 & 323.83 & 778.17 & 123.06 & 637.66 & 383.43 & 151.48 & 108.94 & 50.16 \\
671.49 & 183.16 & 257.44 & 727.23 & 291.27 & 101.15 & 376.42 & 163.40 & 141.38 & 700.74 \\
262.90 & 353.24 & 422.11 & 43.93 & 590.48 & 212.13 & 303.90 & 506.60 & 530.55 & 177.25 \\
\hline
\end{tabular}

Table 13: Dataset 2 (Breaking strength of jute fiber of gauge length $20 \mathrm{~mm}$ ).

\begin{tabular}{cccccccccc}
\hline 71.46 & 419.02 & 284.64 & 585.57 & 456.60 & 113.85 & 187.85 & 688.16 & 662.66 & 45.58 \\
578.62 & 756.70 & 594.29 & 166.49 & 99.72 & 707.36 & 765.14 & 187.13 & 145.96 & 350.70 \\
547.44 & 116.99 & 375.81 & 581.60 & 119.86 & 48.01 & 200.16 & 36.75 & 244.53 & 83.55 \\
\hline
\end{tabular}

Treating these two datasets as universal, we implemented both two-stage and purely sequential procedures, drawing observations $(X, Y)$ from the full set of data as needed. To obtain the fixed-width $2 d$ asymptotic confidence interval for parameter $R$, we carried out a single run under both procedures. Tables 14-15 provide the results derived from implementing the stopping rules from (3.1) and (4.1) respectively, when the initial sample size $m=5,10, \alpha=0.05$ and $d=0.3,0.2,0.15$ are chosen arbitrarily. Under both methodologies, the final estimators $\hat{R}_{N_{m}}$ and $\hat{R}_{N_{d}}$ tended to get closer to their exact value $\hat{R}=0.482$ obtained from the full data as the initial sample size $m$ increases. Both estimators $\hat{R}_{N_{m}}$ and $\hat{R}_{N_{d}}$ are less than 0.5 . It means that jute fibers of gauge length of 20 $\mathrm{mm}$ do not endure more than jute fibers of gauge length of $10 \mathrm{~mm}$. 
Table 14: An illustration with breaking strength of jute fiber of gauge length $10 \mathrm{~mm}, 20$ $\mathrm{mm}$, with $d=0.3,0.2,0.15, m=5,10$ and $\alpha=0.05$ using the two-stage procedure.

\begin{tabular}{|c|c|c|}
\hline$d$ & $m=5$ & $m=10$ \\
\hline 0.3 & $\begin{array}{l}\text { Pilot Data: } \\
X: 50.16,108.94,163.40,376.42,637.66 \\
Y: 594.29,113.85,756.70,36.75,48.01, \\
\bar{X}_{5}=418.976, \bar{Y}_{5}=490.518 \\
\longrightarrow N_{m}=6 \\
\text { Second Stage Data Size: } \\
X: 530.55 \\
Y: 662.66 \\
\bar{X}_{N_{m}}=418.976, \bar{Y}_{N_{m}}=490.518 \\
\longrightarrow \widehat{R}_{N_{m}}=0.5390 \\
\left.\widehat{(R}_{N_{m}}-d, \widehat{R}_{N_{m}}+d\right)=(0.239,0.839) .\end{array}$ & $\begin{array}{l}\text { Pilot Data: } \\
X: 727.23,163.4,637.66,123.06,50.16, \\
671.49,353.24,700.74,212.13,257.44 \\
Y: 200.16,145.96,350.70,578.62,187.13, \\
756.70,36.75,375.81,113.85,166.49 \\
\bar{X}_{10}=389.655, \bar{Y}_{10}=291.217 \\
\longrightarrow N_{m}=10 \\
\text { Second Stage Data Size: } \\
\text { Pilot Data is enough. } \\
\bar{X}_{N_{m}}=389.655, \bar{Y}_{N_{m}}=291.217 \\
\longrightarrow \widehat{R}_{N_{m}}=0.4277 \\
\left.\widehat{(R}_{N_{m}}-d, \widehat{R}_{N_{m}}+d\right)=(0.1277,0.7277) .\end{array}$ \\
\hline 0.2 & $\begin{array}{l}\text { Pilot Data: } \\
X: 303.90,212.13,291.27,693.73,383.43 \\
Y: 116.99,45.58,581.60,707.36,119.86 \\
\bar{X}_{5}=376.892, \bar{Y}_{5}=314.278 \\
\longrightarrow N_{m}=12 \\
\text { Second Stage Data Size: } \\
X: 151.48,727.23,637.66,353.24,530.55 \\
177.25,101.15 \\
Y: 662.66,375.81,688.16,145.96,48.01 \\
284.64,113.85 \\
\bar{X}_{N_{m}}=380.2517, \bar{Y}_{N_{m}}=324.2067 \\
\longrightarrow \widehat{R}_{N_{m}}=0.4602 \\
\left.\widehat{(R}_{N_{m}}-d, \widehat{R}_{N_{m}}+d\right)=(0.2602,0.6602)\end{array}$ & $\begin{array}{l}\text { Pilot Data: } \\
X: 212.13,704.66,727.23,303.9,530.55, \\
323.83,123.06,693.73,141.38,506.6 \\
Y: 48.01,581.60,765.14,244.53,284.64, \\
116.99,350.70,662.66,756.70,113.85 \\
\bar{X}_{10}=426.707, \bar{Y}_{10}=392.482 \\
\longrightarrow N_{m}=12 \\
\text { Second Stage Data Size: } \\
X: 506.60,212.13 \\
Y: 350.70,116.99 \\
\bar{X}_{N_{m}}=415.4833, \bar{Y}_{N_{m}}=366.0425 \\
\longrightarrow \widehat{R}_{N_{m}}=0.4684 \\
\left.\widehat{(R}_{N_{m}}-d, \widehat{R}_{N_{m}}+d\right)=(0.2684,0.6684) .\end{array}$ \\
\hline 0.15 & $\begin{array}{l}\text { Pilot Data: } \\
X: 257.44,323.83,303.9,506.6,123.06 \\
Y 578.62,166.49,244.53,36.75,113.85 \\
\bar{X}_{5}=302.966, \bar{Y}_{5}=228.048 \\
\longrightarrow N_{m}=21 \\
\text { Second Stage Data Size: } \\
X: 530.55,422.11,323.83,700.74,123.06, \\
\text { 108.94, 183.16, 163.40, 590.48, 177.25, } \\
778.17,727.23,101.15,212.13,50.16 \\
262.9 \\
Y: 594.29,113.85,36.75,145.96,375.81 \\
187.85,765.14,547.44,116.99,419.02 \\
71.46,662.66,99.72,581.60,244.53 \\
48.01 \\
\bar{X}_{N_{m}}=331.909, \bar{Y}_{N_{m}}=292.92 \\
\longrightarrow \widehat{R}_{N_{m}}=0.4688 \\
\left(\widehat{R}_{N_{m}}-d, \widehat{R}_{N_{m}}+d\right)=(0.3188,0.6188) .\end{array}$ & $\begin{array}{l}\text { Pilot Data: } \\
X: 141.38,530.55,376.42,353.24,693.73, \\
637.66,43.93,291.27,212.13,383.43 \\
Y 119.86,765.14,350.70,200.16,375.81, \\
116.99,662.66,244.53,707.36,166.49 \\
\bar{X}_{10}=366.374, \bar{Y}_{10}=370.97 \\
\longrightarrow N_{m}=22 \\
\text { Second Stage Data Size: } \\
X: 506.60,177.25,43.93,108.94,183.16, \\
671.49,353.24,141.38,727.23,50.16, \\
778.17,123.06 \\
Y: 200.16,45.58,113.85,594.29,585.57, \\
71.46,662.66,419.02,116.99,166.49, \\
284.64,48.01 \\
\bar{X}_{N_{m}}=342.1977, \bar{Y}_{N_{m}}=319.0191 \\
\widehat{R}_{N_{m}}=0.4825 \\
\left(\widehat{R}_{N_{m}}-d, \widehat{R}_{N_{m}}+d\right)=(0.3325,0.6325) .\end{array}$ \\
\hline
\end{tabular}


Table 15: An illustration with breaking strength of jute fiber of gauge length $10 \mathrm{~mm}, 20$ mm with $d=0.3,0.2,0.15, m=5,10$ and $\alpha=0.05$ using the purely sequential procedure.

\begin{tabular}{|c|c|c|}
\hline$d$ & $m=5$ & $m=10$ \\
\hline 0.3 & $\begin{array}{l}\text { Pilot Data: } \\
X: 141.38,101.15,671.49,163.40,151.48 \\
Y: 187.85,688.16,48.01,284.64,547.44 \\
\text { Samples } \\
X: 257.44 \\
Y: 83.55 \\
\vec{X}_{N_{d}}=247.723, N_{d}=6 \\
\widehat{R}_{N_{d}}=0.553 \\
\left(\widehat{R}_{N_{d}}-d, \widehat{R}_{N_{d}}+d\right)=(0.253,0.853) \\
\end{array}$ & $\begin{array}{l}\text { Pilot Data: } \\
X: 671.49,323.83,506.60,123.06,151.48, \\
\text { 183.16, 177.25, 163.40, 637.66, 376.42 } \\
Y: 187.85,113.85,585.57,707.36,284.64 \\
\text { 99.72, 145.96, 166.49, 419.02, 375.81 } \\
\text { Samples } \\
\text { Initial Sample is sufficient. } \\
\longrightarrow N_{d}=10 \\
\bar{X}_{N_{d}}=331.435, \bar{Y}_{N_{d}}=308.627 \\
\widehat{R}_{N_{d}}=0.482 \\
\left.\widehat{(R}_{N_{d}}-d, \widehat{R}_{N_{d}}+d\right)=(0.182,0.782) \\
\end{array}$ \\
\hline 0.2 & $\begin{array}{l}\text { Pilot Data: } \\
X: 700.74,257.44,177.25,212.13,778.17 \\
Y: 166.49,594.29,547.44,200.16,45.58 \\
\text { Samples } \\
X: 671.49,376.42,637.66,123.06,123.06 \\
Y: 119.86,187.85,166.49,99.72,36.75 \\
\longrightarrow N_{d}=10, \bar{X}_{N_{d}}=405.742, \bar{Y}_{N_{d}}=216.463 \\
\widehat{R}_{N_{d}}=0.347 \\
\left(\widehat{R}_{N_{d}}-d, \widehat{R}_{N_{d}}+d\right)=(0.147,0.547)\end{array}$ & $\begin{array}{l}\text { Pilot Data: } \\
X: 778.17,693.73,141.38,727.23,212.13, \\
177.25,108.94,123.06,671.49,506.60 \\
Y: 36.75,48.01,581.60,200.16,350.70, \\
\text { 707.36, 662.66, 594.29, 71.46, 419.02 } \\
\text { Samples } \\
X: 177.25,671.49 \\
Y: 187.85,200.16 \\
\longrightarrow N_{d}=12, \bar{X}_{N_{d}}=415.726, \bar{Y}_{N_{d}}=338.335, \\
\widehat{R}_{N_{d}}=0.448 \\
\left(\widehat{R}_{N_{d}}-d, \widehat{R}_{N_{d}}+d\right)=(0.248,0.648)\end{array}$ \\
\hline 0.15 & $\begin{array}{l}\text { Pilot Data: } \\
X: 43.93,212.13,291.27,422.11,163.40 \\
Y: 48.01,578.62,99.72,200.16,594.29 \\
\text { Samples } \\
X: 303.90,262.90,108.94,671.49,123.06, \\
141.38,177.25,177.25,262.90,212.13, \\
530.55,257.44,704.66,704.66,101.15, \\
506.60,262.90 \\
Y: 688.16,707.36,45.58,688.16,350.70,166.49, \\
456.60,113.85,45.58,36.75,116.99, \\
662.66,244.53,145.96,244.53, \\
284.64,578.62 \\
\longrightarrow N_{d}=22, \bar{X}_{N_{d}}=301.909, \bar{Y}_{N_{d}}=322.634, \\
\widehat{R}_{N_{d}}=0.516 \\
\left(\widehat{R}_{N_{d}}-d, \widehat{R}_{N_{d}}+d\right)=(0.366,0.666) \\
\end{array}$ & $\begin{array}{l}\text { Pilot Data: } \\
X: 383.43,353.24,530.55,151.48,163.40, \\
700.74,212.13,376.42,262.90,257.44 \\
Y: 456.60,48.01,350.70,578.62,113.85, \\
\text { 707.36, 547.44, 244.53, 45.58, 116.99 } \\
\text { Samples } \\
X: 257.44,257.44,693.73,50.16,671.49, \\
50.16,163.40,376.42,704.66,183.16, \\
506.60,671.49 \\
Y: 585.57,187.13,578.62,113.85,284.64, \\
187.85,578.62,83.55,585.57,71.46, \\
375.81,99.72 \\
\longrightarrow N_{d}=22, \bar{X}_{N_{d}}=362.630, \bar{Y}_{N_{d}}=315.548, \\
\widehat{R}_{N_{d}}=0.465 \\
\left.\widehat{(R}_{N_{d}}-d, \widehat{R}_{N_{d}}+d\right)=(0.315,0.615) \\
\end{array}$ \\
\hline
\end{tabular}

To show that the coverage frequency is correspondingly higher than 0.95 , we carried out 100 runs under both procedures for each case of Tables 14-15. We found, $\hat{R}_{N_{d}}$ for purely sequential and $\hat{R}_{N_{m}}$ for two-stage sequential procedures, then constructed $2 d$ fixed-width confidence interval $\left(\hat{R}_{N_{d}}^{(i)}-d, \hat{R}_{N_{d}}^{(i)}+d\right)$ and $\left(\hat{R}_{N_{m}}^{(i)}-d, \hat{R}_{N_{m}}^{(i)}+d\right)$ for purely and 
two-stage sequential procedures, respectively, for the $i^{\text {th }}$ run. Define $Z_{N_{d}}^{(i)}$ such that

$$
Z_{N_{d}}^{(i)}= \begin{cases}1 & \hat{R} \in\left(\hat{R}_{N_{d}}^{(i)}-d, \hat{R}_{N_{d}}^{(i)}+d\right) \\ 0 & \text { O.W. }\end{cases}
$$

Similarly define $Z_{N_{m}}^{(i)}$ in which $N_{m}$ is substituted for $N_{d}$. The coverage probability (frequency) is $\sum_{i=1}^{100} z_{N_{d}}^{(i)} / 100$ for purely sequential or $\sum_{i=1}^{100} z_{N_{m}}^{(i)} / 100$ for two-stage sequential procedures. The results of coverage frequency for purely and two-stage sequential procedures are summarized in Table 16. It can be seen that for all the cases, the coverage frequencies are higher than 0.95 .

Table 16: Coverage frequency for breaking strength of jute fiber of gauge length $10 \mathrm{~mm}$, $20 \mathrm{~mm}$ with 100 runs.

\begin{tabular}{lrccc}
\hline procedures & $m$ & $d=0.3$ & $d=0.2$ & $\mathrm{~d}=0.15$ \\
\hline \multirow{2}{*}{ purely sequential } & 5 & 0.99 & 0.98 & 0.98 \\
& 10 & 1.00 & 0.99 & 0.98 \\
\hline \multirow{2}{*}{ two-stage sequential } & 5 & 0.99 & 0.97 & 0.96 \\
& 10 & 1.00 & 0.98 & 0.98 \\
\hline
\end{tabular}

\section{Conclusion}

Two-stage and purely sequential procedures have been investigated to construct the fixed-width $2 d$ confidence interval for reliability parameter under the stress-strength model in the exponential two-sample problem. Approximation of exact distribution of purely sequential procedure, and the exact distribution of two-stage sequential procedure, and their characteristics have also been discussed for the different pilot sample sizes. The accuracy of our method to approximate the distribution of purely sequential procedure has been assessed by using simulation results. Both of these procedures have different routes, merits, and demerits. A two-stage technique is often easy to implement, whereas a purely sequential methodology is proved to be more accurate. One should, however, balance any logistical concerns, such as feasibility and cost.

In the current research, it has been assumed that the samples taken from two 
populations are of the same size. However, it might not be the case in reality. So, the MLE of $R$ can be written as,

$$
\widehat{R}_{n_{1}, n_{2}}=\frac{\bar{Y}_{n_{2}}}{\bar{Y}_{n_{2}}+\bar{X}_{n_{1}}},
$$

where $n_{1}$ is the size of the sample taken from the stress $(X)$ population, and $n_{2}$ is the size of the sample from the strength $(Y)$ population. Therefore, defining a stopping rule to find optimal $n_{1}^{*}, n_{2}^{*}$ for constructing a fixed-width $2 d$ confidence interval with confidence

at least $1-\alpha$ for $R$ based on $\hat{R}_{n_{1}, n_{2}}$, is yet to be studied and deserves a comprehensive research. Moreover, the exact distribution and asymptotic second-order properties for the proposed purely sequential procedure in this research, are yet to be addressed.

\section{Acknowledgements}

The authors would like to thank the anonymous reviewers for their helpful and constructive comments which significantly contributed to improving the final version of the paper. They also would like to thank the Associate Editors for their generous comments and support during the review process.

\section{References}

Awad, A. M., Azzam, M. M., and Hamdan, M. A. (1981), Some inference results on $\operatorname{Pr}(X<Y)$ in the bivariate exponential model. Communications in Statistics-Theory and Methods, 10, 2515-2525.

Bandyopadhyay, U., Das, R., and Biswas, A. (2003), Fixed width confidence interval of $P(X<Y)$ in partial sequential sampling scheme. Sequential Analysis, 22, 75-93.

Bapat, S. R. (2018), Purely sequential fixed accuracy confidence intervals for $P(X<Y)$ under bivariate exponential models. American Journal of Mathematical and Management Sciences, 37, 386-400.

Beg, M. A. (1980), On the estimation of $\operatorname{Pr}(Y<X)$ for the two-parameter exponential distribution. Metrika, 27, 29-34.

Birnbaum, Z. W. (1956), On a use of the mann-whitney statistic. In Proceedings of the Third Berkeley Symposium on Mathematical Statistics and Probability, Ed. J. Neyman. Berkeley: University of California, 13-17. 
Birnbaum, Z. W. and McCarty, R. C. (1958), A distribution-free upper confidence bound for $P(Y<X)$, based on independent samples of $X$ and $Y$. The Annals of Mathematical Statistics, 29, 558-562.

Chao, A. (1982), On comparing estimators of $\operatorname{Pr}(Y<X)$ in the exponential case. IEEE Transactions on Reliability, 31, 389-392.

Chiodo, E. (2014), Model robustness analysis of a bayes stress-strength reliability estimation with limited data. In 2014 International Symposium on Power Electronics, Electrical Drives, Automation and Motion. New York: IEEE, 1140-1145.

Cramer, E. and Kamps, U. (1997), The umvue of $P(X<Y)$ based on type-II censored samples from weinman multivariate exponential distributions. Metrika, 46, 93-93.

Domma, F. and Giordano, S. (2013), A copula-based approach to account for dependence in stress-strength models. Statistical Papers, 54, 807-826.

Enis, P. and Geisser, S. (1971), Estimation of the probability that $Y<X$. Journal of the American Statistical Association, 66, 162-168.

Ferguson, T. (1996). A course in large sample theory. New York: Chapman and Hall.

Freund, J. E. (1961), A bivariate extension of the exponential distribution. Journal of the American Statistical Association, 56, 971-977.

Govindarajulu, Z. (1974), Fixed-width confidence intervals for $P(X<Y)$. In Reliability and biometry: statistical analysis of lifelength, Eds. E. F. Proschan and R. J. Serfling. Philadelphia: SIAM, 747-757.

Govindarajulu, Z. (2004), Sequential Estimation. Singapore: World Scientific.

Johnson, R. A. (1988), 3 stress-strength models for reliability. In Quality Control and Reliability, Eds. P. R. Krishnaiah and C. R. Rao. Amsterdam: Elsevier, 27-54.

Kao, E. (1997), An Introduction to Stochastic Processes. Scituate :Duxbury.

Kelley, G. D., Kelley, J. A., and Schucany, W. R. (1976), Efficient estimation of $P(Y<X)$ in the exponential case. Technometrics, 18, 359-360.

Kotz, S., Lumel'skii, I., and Pensky, M. (2003), The Stress-strength Model and Its Generalizations: Theory and Applications. Singapore: World Scientific. 
Kundu, D. and Gupta, R. D. (2006), Estimation of $P(Y<X)$ for weibull distributions. IEEE Transactions on Reliability, 55, 270-280.

Lim, D. L., Isogai, E., and Uno, C. (2004), Two-sample fixed width confidence intervals for a function of exponential scale parameters. Far East Journal of Theoretical Statistics, 14, 215-227.

Mahmoudi, E., Khalifeh, A., and Nekoukhou, V. (2018), Minimum risk sequential point estimation of the stress-strength reliability parameter for exponential distribution. Sequential Analysis. doi: 10.1080/07474946.2019.1649347.

Marshall, A. W. and Olkin, I. (1967), A generalized bivariate exponential distribution. Journal of Applied Probability, 4, 291-302.

Mirjalili, M., Torabi, H., Nadeb, H., and Bafekri. F., S. (2016), Stress-strength reliability of exponential distribution based on type-I progressively hybrid censored samples. Journal of Statistical Research of Iran, 13, 89-105.

Mukhopadhyay, N. and Zhuang, Y. (2016), On fixed-accuracy and bounded accuracy confidence interval estimation problems in fisher's "nile" example. Sequential Analysis, 35, 516-535.

Nadarajah, S. and Kotz, S. (2006), Reliability for some bivariate exponential distributions. Mathematical Problems in Engineering, 2006, 1-14.

Patowary, A. N., Hazarika, J., and Sriwastav, G. L. (2013), Interference theory of reliability: a review. International Journal of System Assurance Engineering and Management, 4, 146-158.

Sathe, Y. and Shah, S. (1981), On estimating $P(X>Y)$ for the exponential distribution. Communications in Statistics - Theory and Methods, 10, 39-47.

Stein, C. (1945), A two-sample test for a linear hypothesis whose power is independent of the variance. The Annals of Mathematical Statistics, 16, 243-258.

Tong, H. (1974), A note on the estimation of $\operatorname{Pr}(Y<X)$ in the exponential case. Technometrics, 16, 625-625.

Xia, Z., Yu, J., Cheng, L., Liu, L., and Wang, W. (2009), Study on the breaking strength of jute fibres using modified weibull distribution. Composites Part A: Applied Science and Manufacturing, 40, 54-59. 